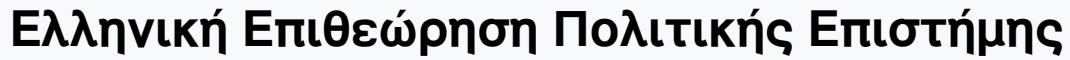

Tó 37 (2011)

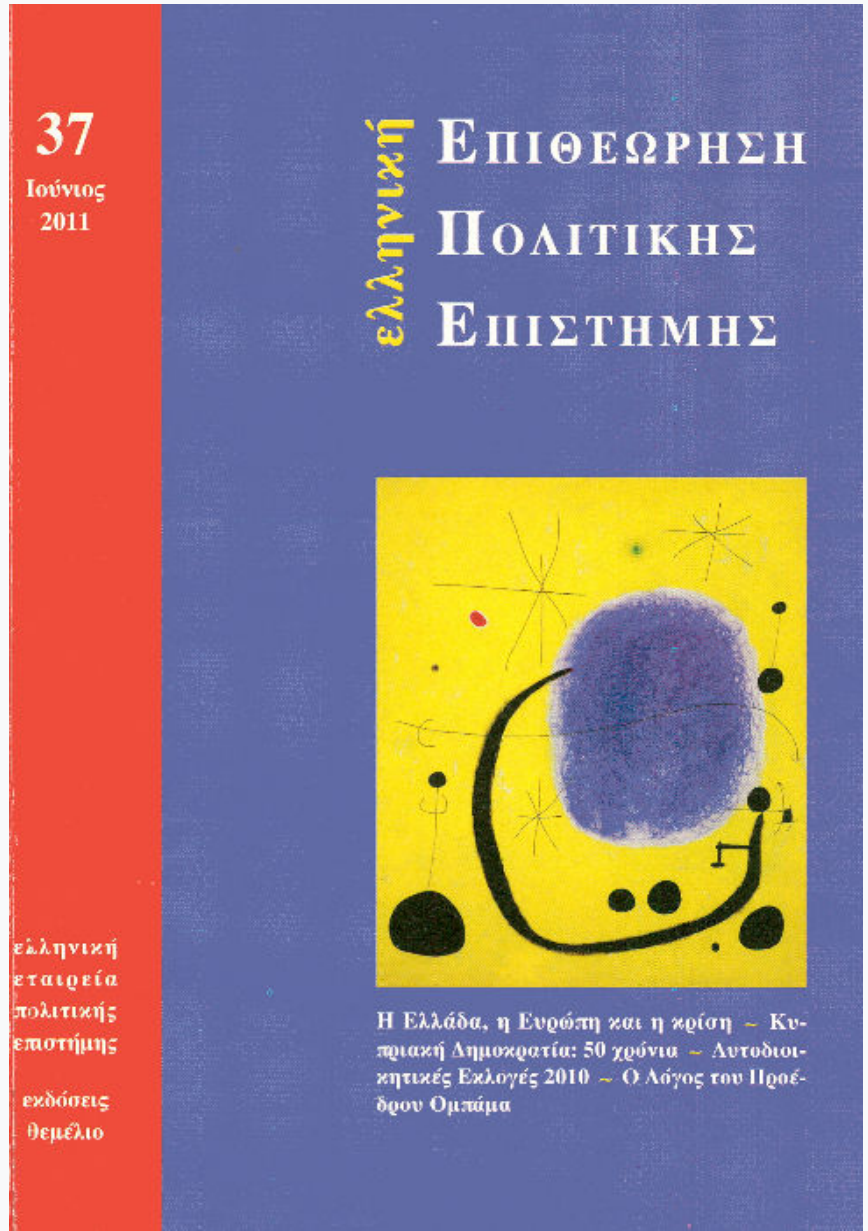

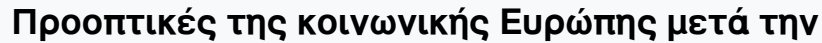 кpíon}

\section{Mávoc Matoayyávis}

doi: $10.12681 / \mathrm{hpsa} .14517$

Copyright @ 2017, Mávoc Matoayyávnৎ

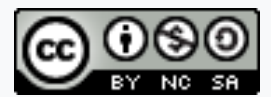

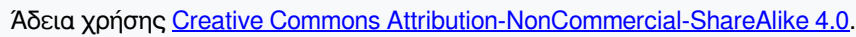

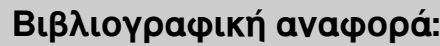

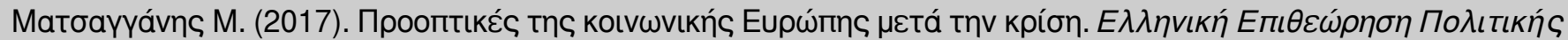

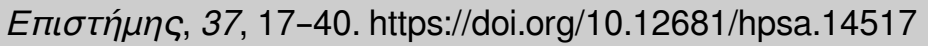




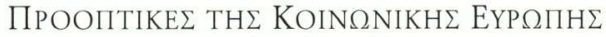

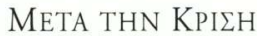
Mávos Matoayyávns:

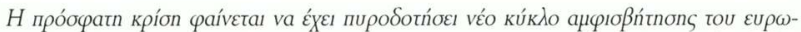

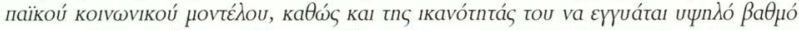

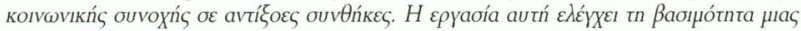

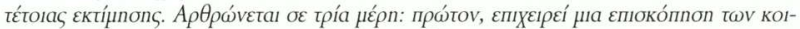

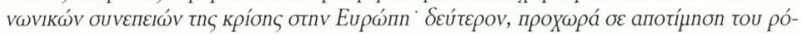

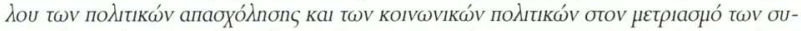

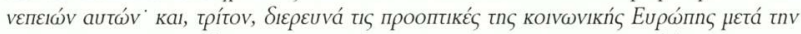

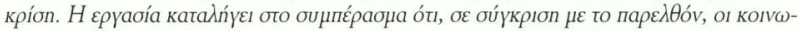

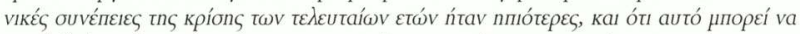

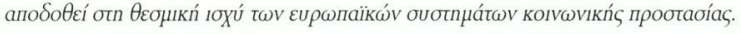

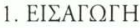

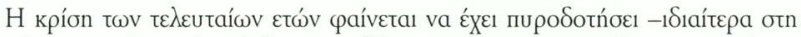

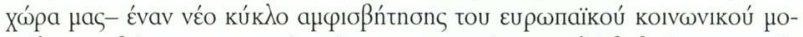

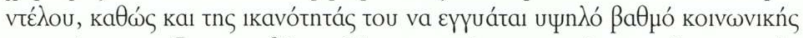

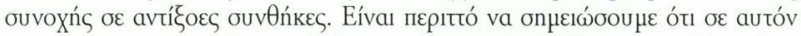

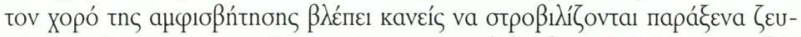

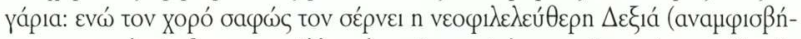

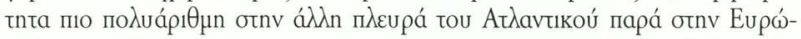

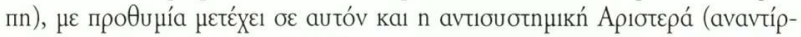

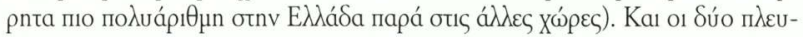

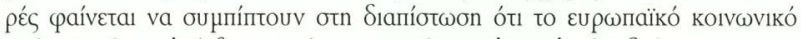

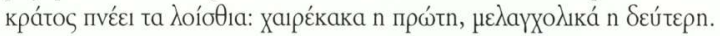

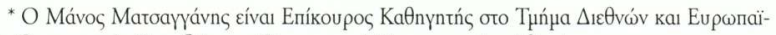

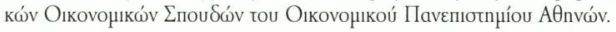




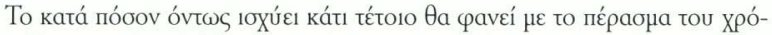

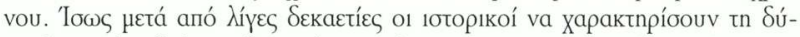

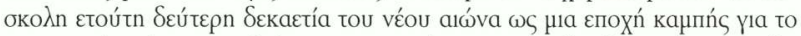

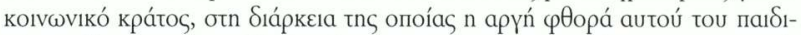

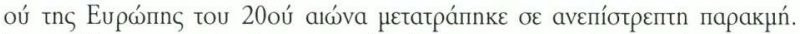

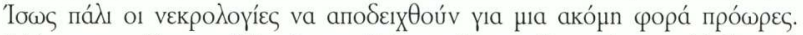

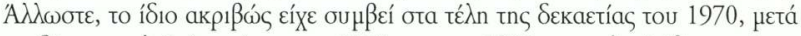

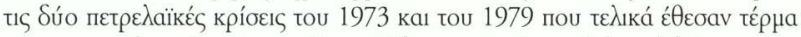

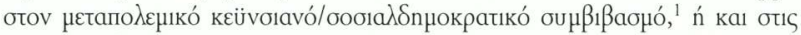

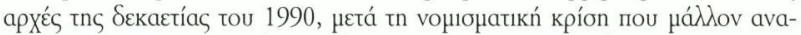

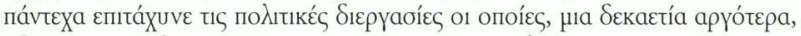

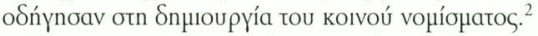

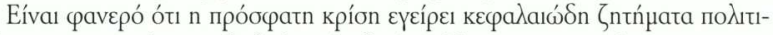

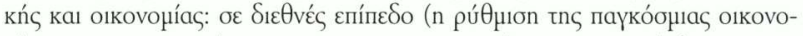

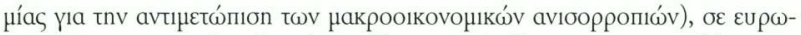

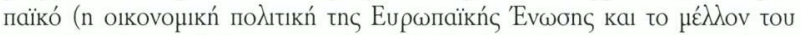

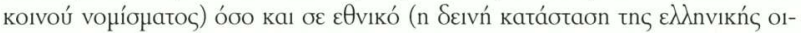

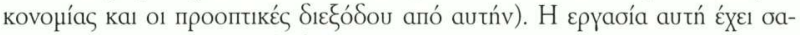

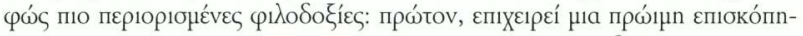

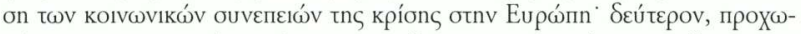

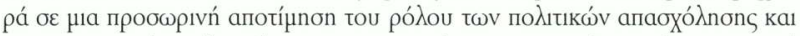

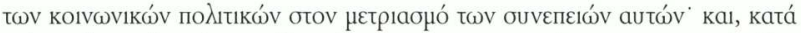

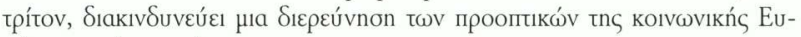

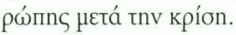

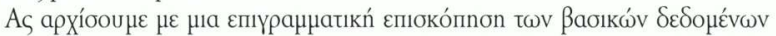

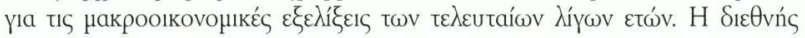

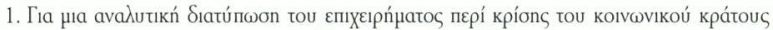

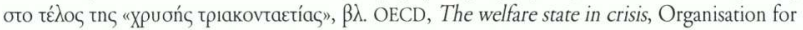

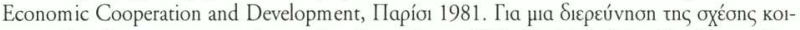

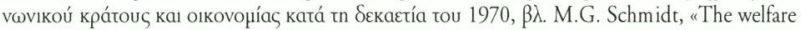
state and the economy in periods of economic crisis: a comparative study of twenty-three OECD nations", European Journal of Political Research, tóp. 11, тx. 1, 1983, o. 1-26.

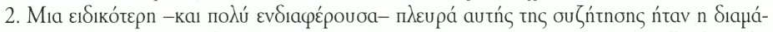

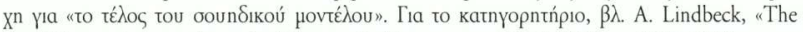
Swedish experiment», Journal of Economic Literature тХ. XXXV, 1997, б. 1273-1319 кал ү1а

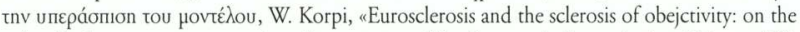
role of values among economic policy experts", The Economic Journal, tóp. 106, тx. 439, 1996, о. 1727-1746. 


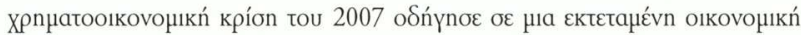

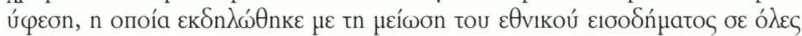

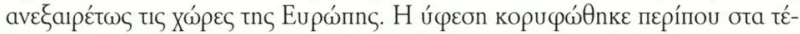

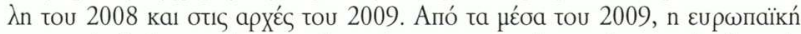

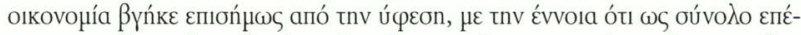

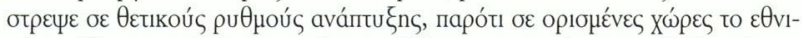

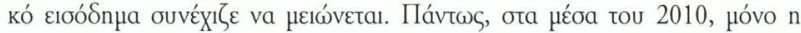

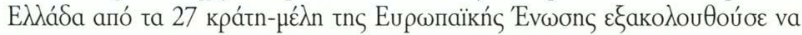

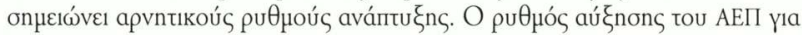

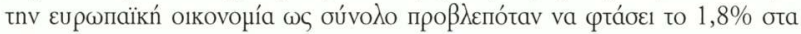

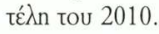

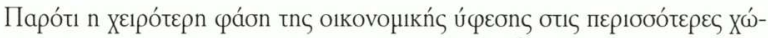

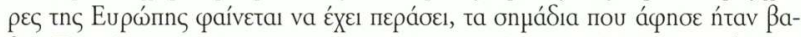

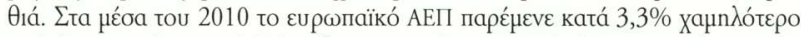

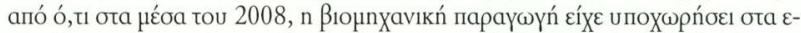

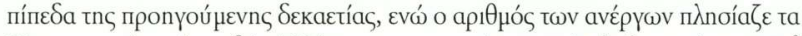

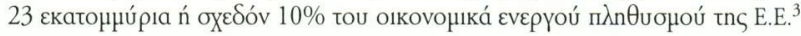

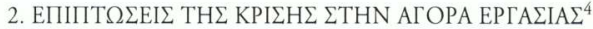

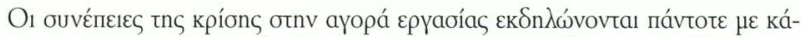

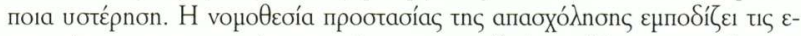

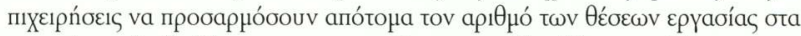

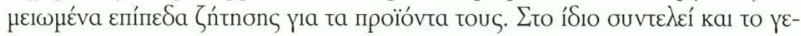

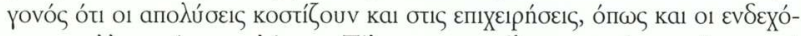

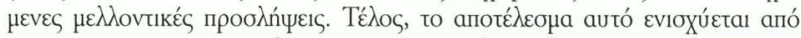

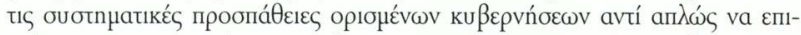

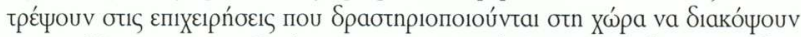

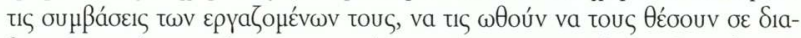

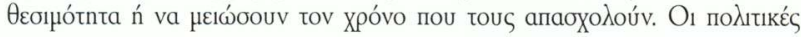

3. B $\mathrm{A}$. EC, Directorate-General for Employment, Social Affairs and Equal Opportunities, Employment in Europe 2010, European Commission, Bpu द́̉̇des 2010.

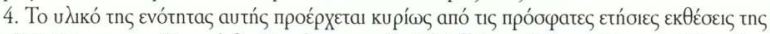
E.E. (EC, Directorate-General for Employment, Social Affairs and Equal Opportunities, Employment in Europe 2010, ó.п.) kal tou OOZA (OECD, Employment outlook: moving beyond the jobs crisis. Organisation for Economic Cooperation and Development, Парі́ø 2010). 


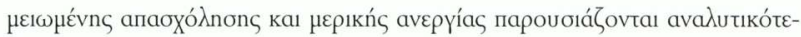
pa orn ouvéxeia.

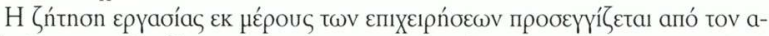

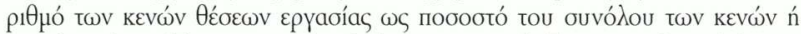

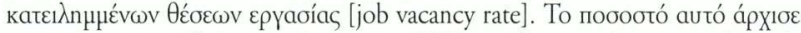

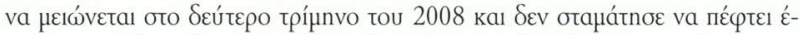

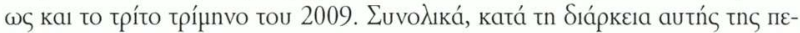

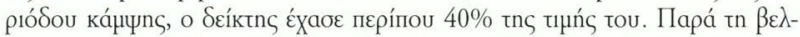

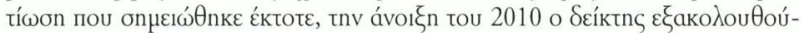

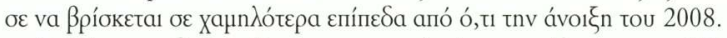

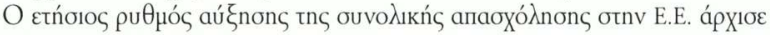
va пе́

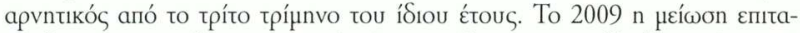

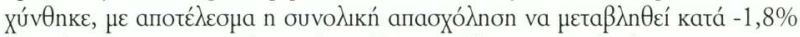

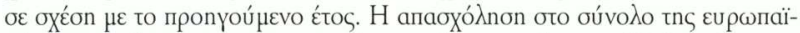

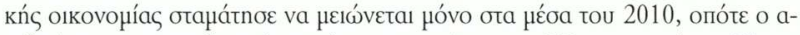

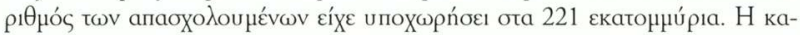

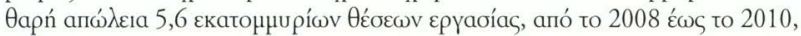

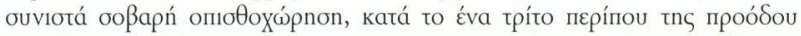

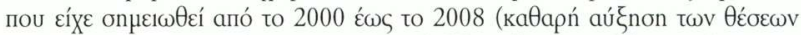

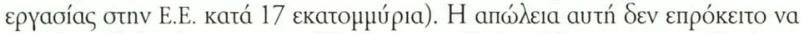

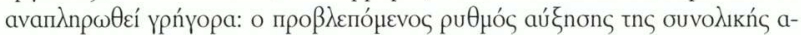

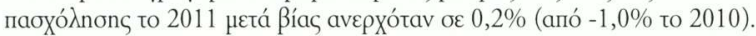

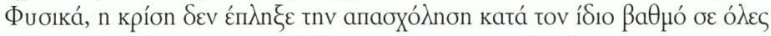

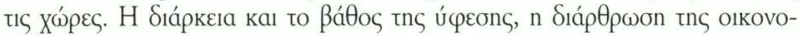

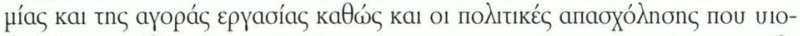

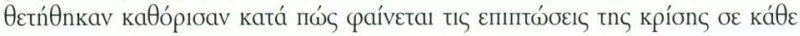

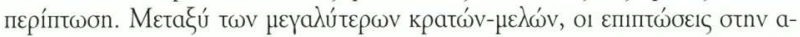

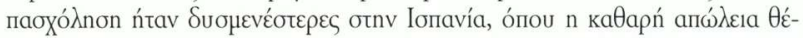

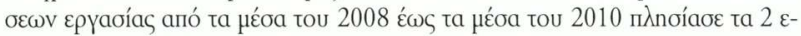

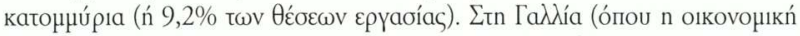

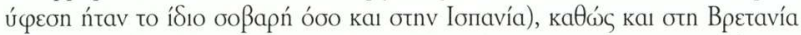

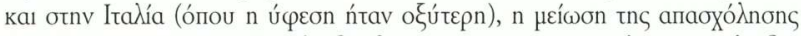

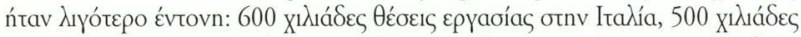

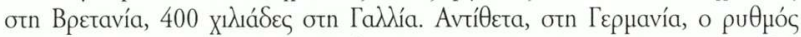

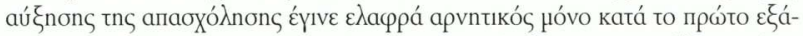

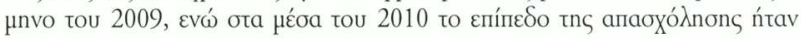




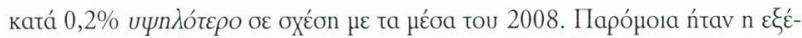

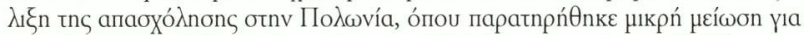

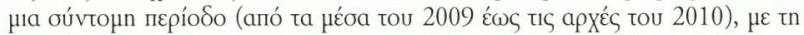

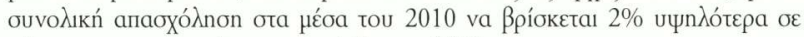

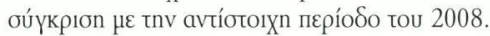

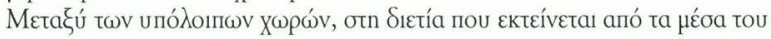

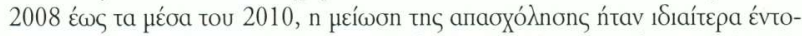

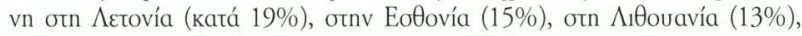

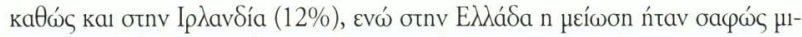

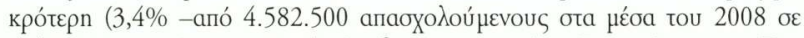

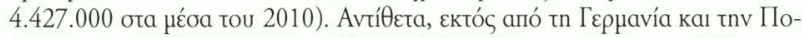

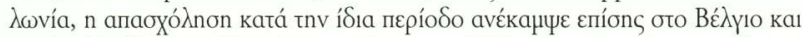

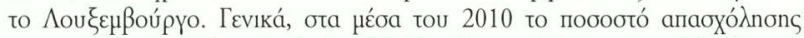

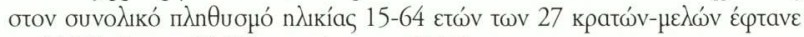

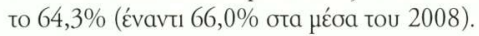

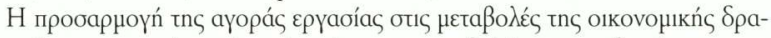

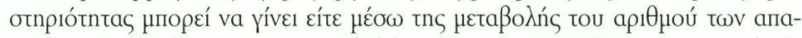

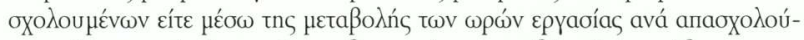

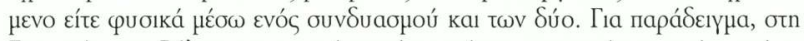

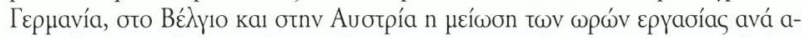

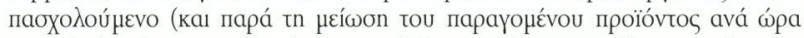

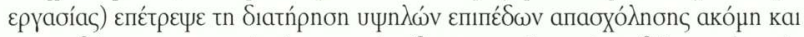

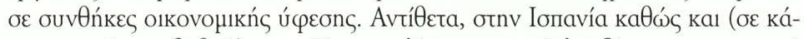

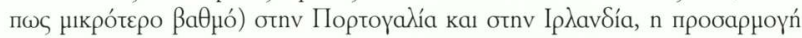

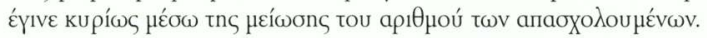

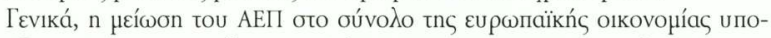

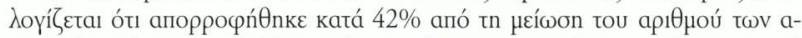

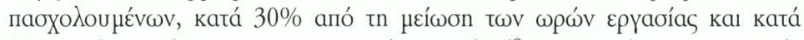

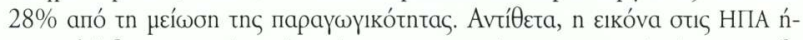

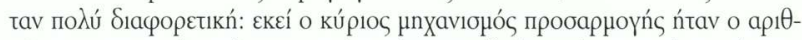

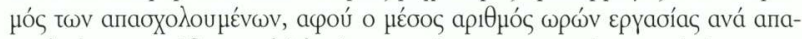

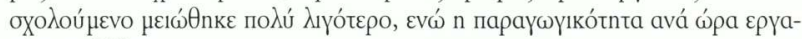
oíac au द́ंӨnke.

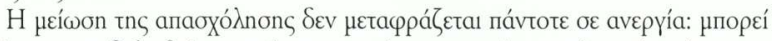

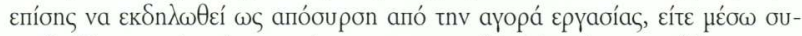

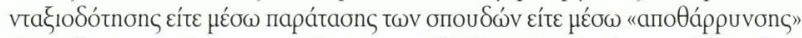

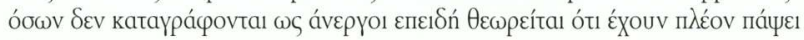




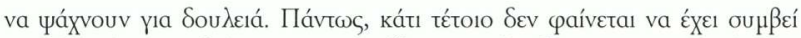

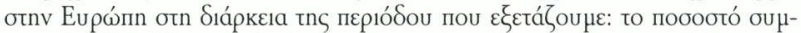

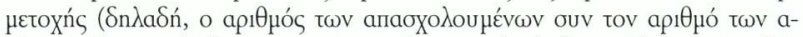

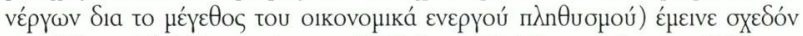
a

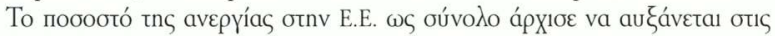

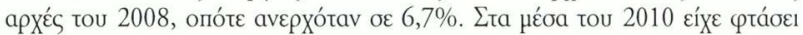

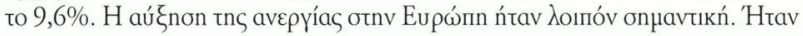

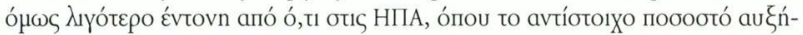

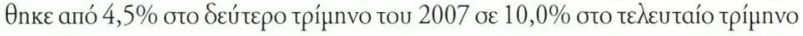

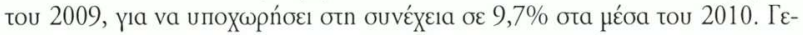

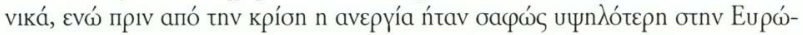

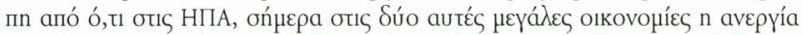

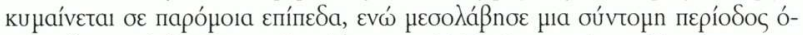

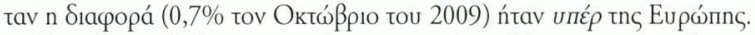

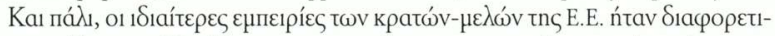

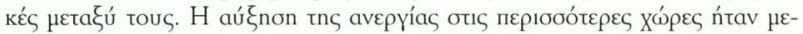

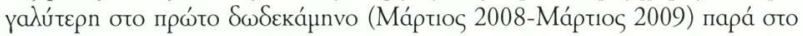

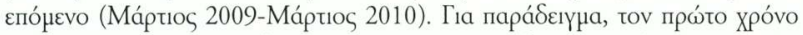

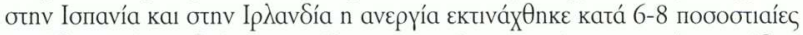

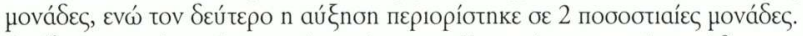

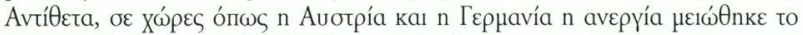

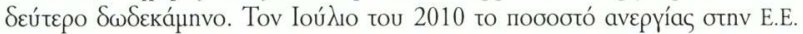

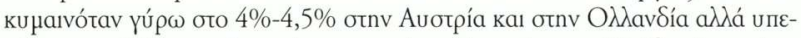

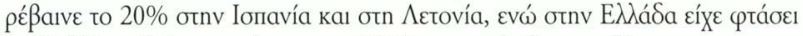

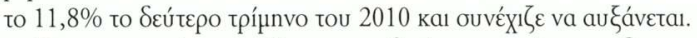

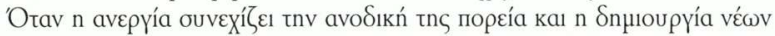

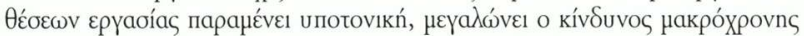

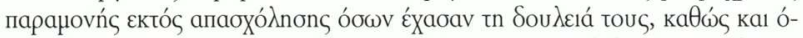

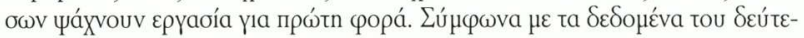

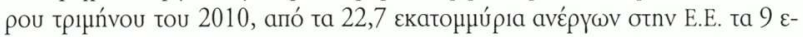

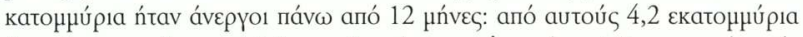

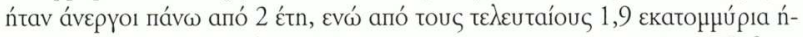

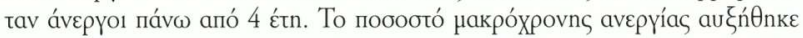

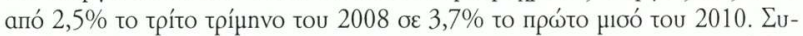

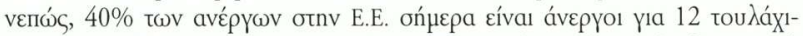

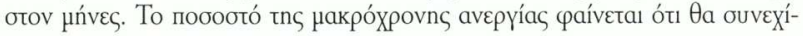




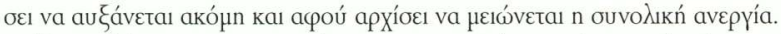

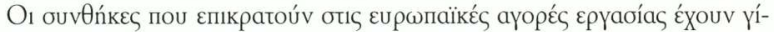

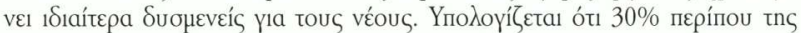

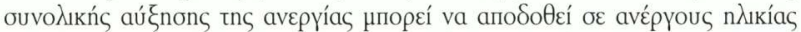

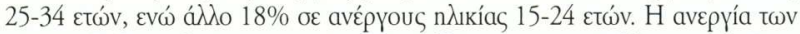

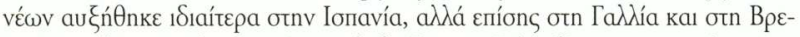

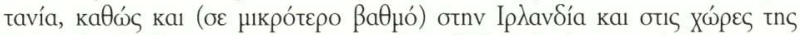

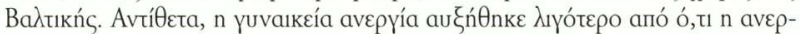

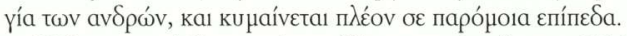

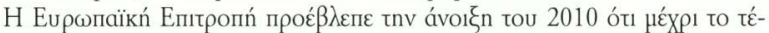

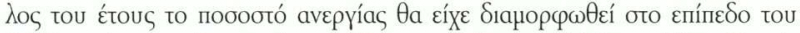

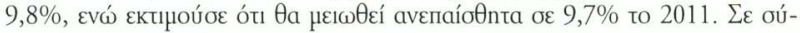

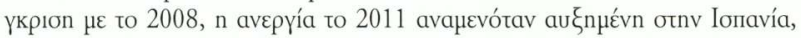

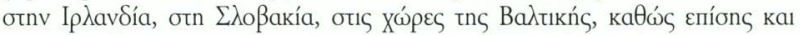

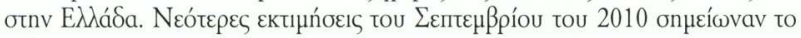

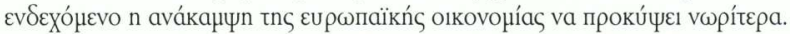

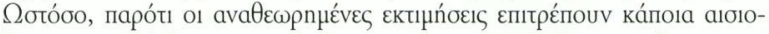

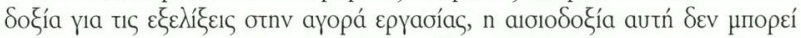

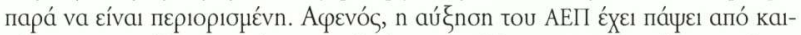

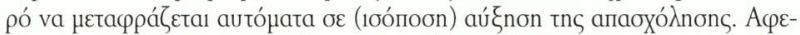

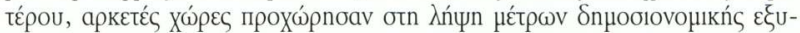

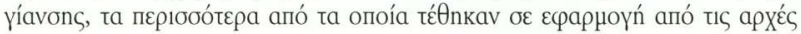

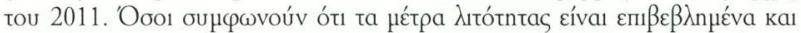

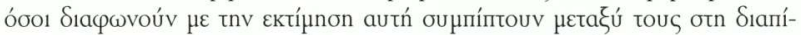

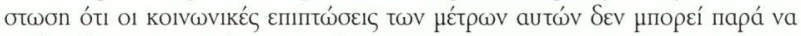

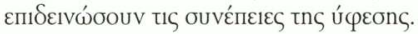

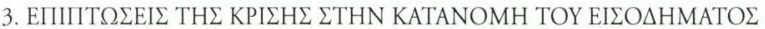

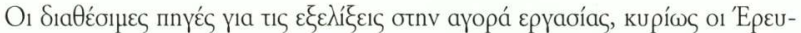

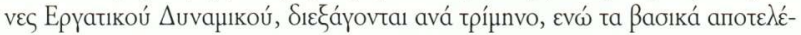

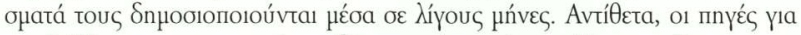

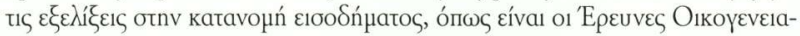

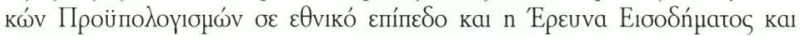

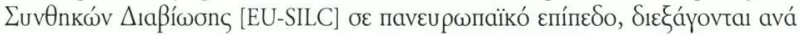

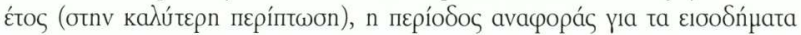




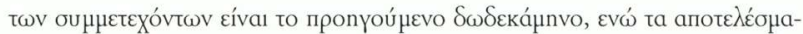

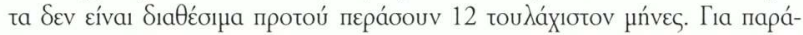

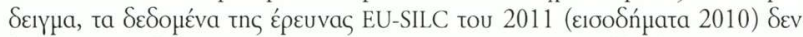

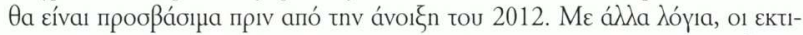

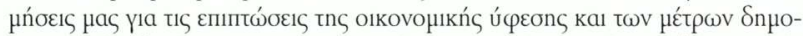

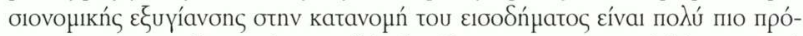

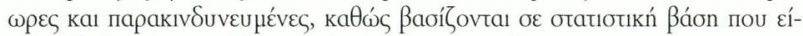

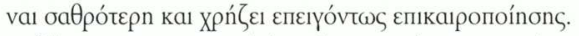

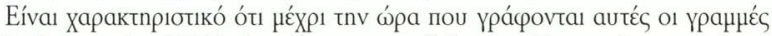

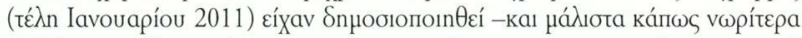

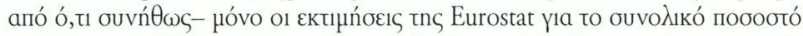

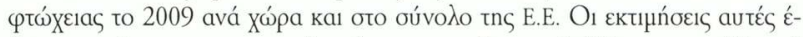

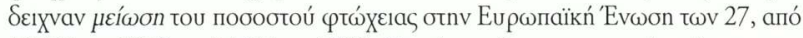

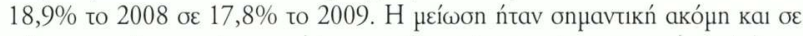

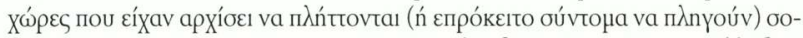
ßара́ апо́ тnv оккоvонıки́ ú

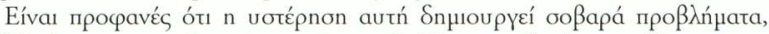

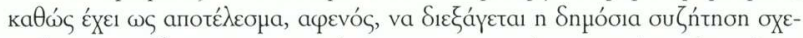

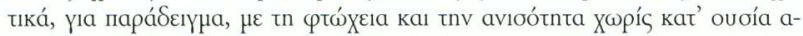
о

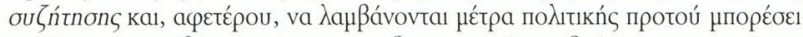

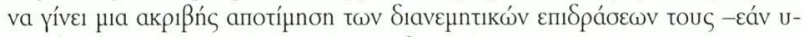

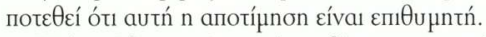

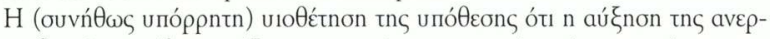

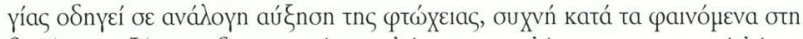

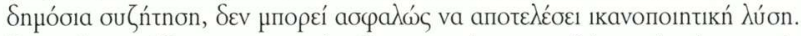

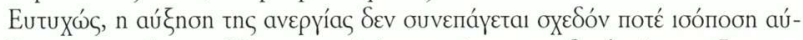

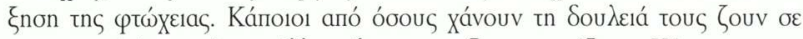

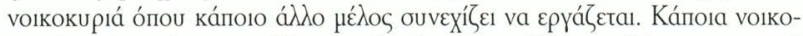

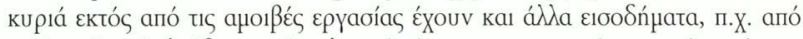

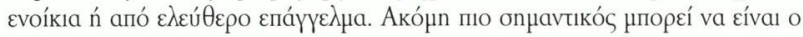

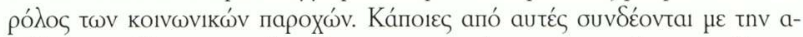

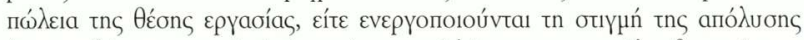

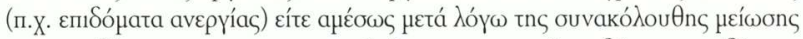

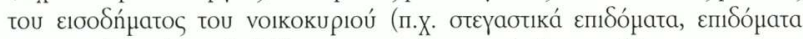

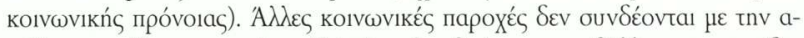

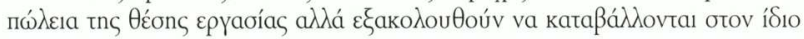




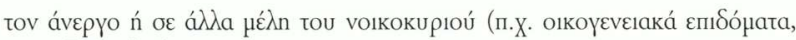

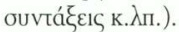

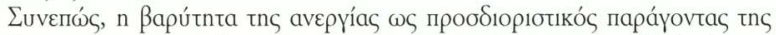

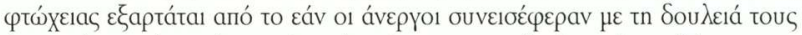

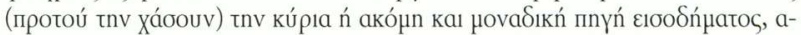

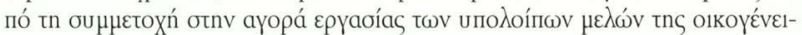

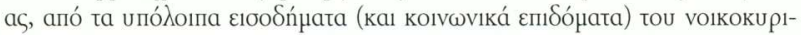

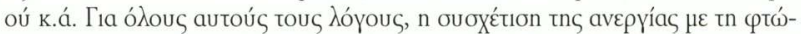

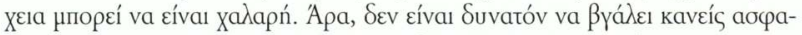

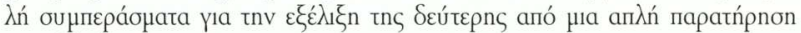

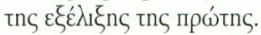

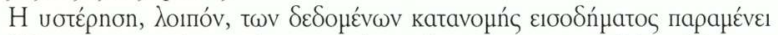

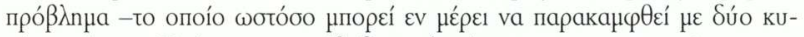

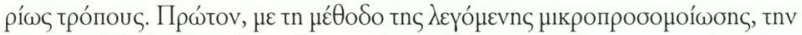

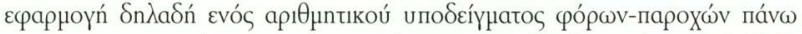

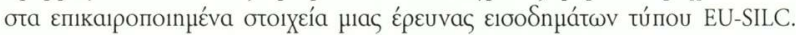

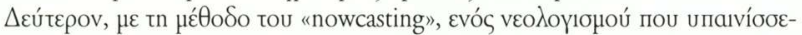

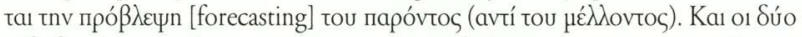

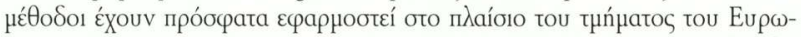

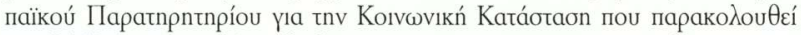

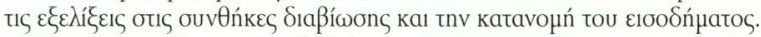

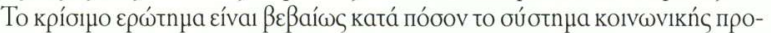

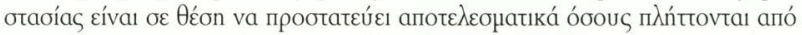

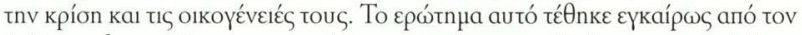

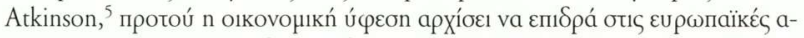

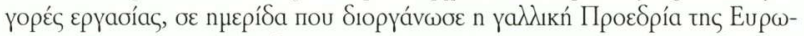

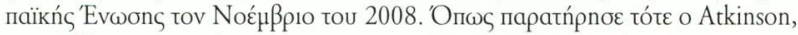

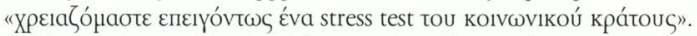

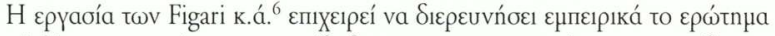

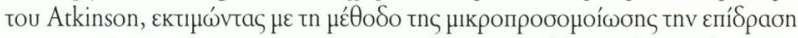

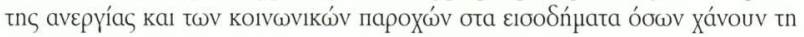

5. A.B. Atkinson, «Stress-testing the welfare state», бто B. Ofstad - O. Bjerkholt - K. Skrede - A. Hylland (впı.), Rettferd og politikk: festskrift til Hilde Bojer pả 70-ärsdagen. Emiliar Forlag, 'Oøגo 2009.

6. F. Figari - A. Salvatori - H. Sutherland, "Economic downturn and stress testing European welfare systems», working paper No. 2010-18, University of Essex: Institute for Social and Economic Research, Essex 2010. 


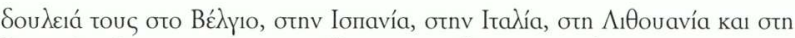

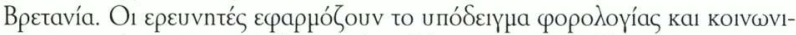

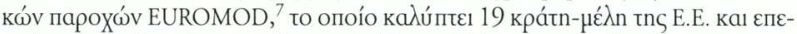

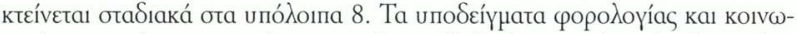

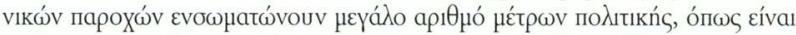

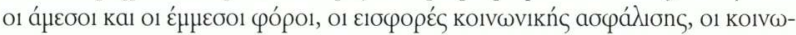

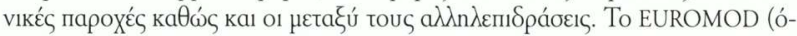

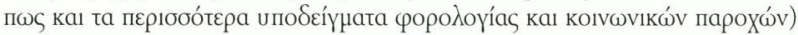

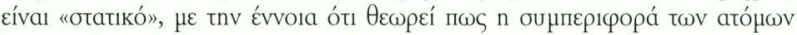

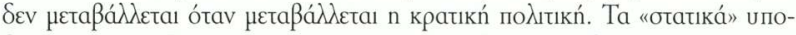

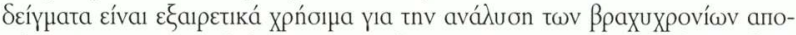

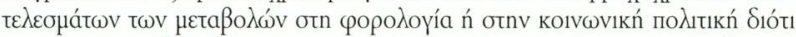

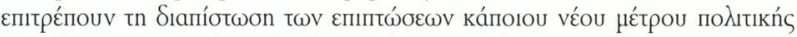

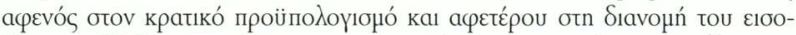

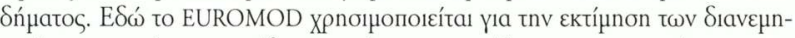

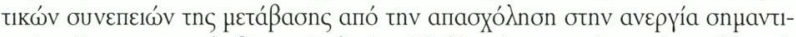

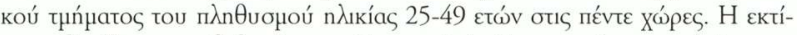

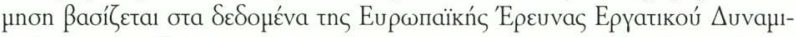

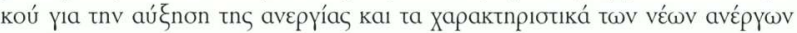

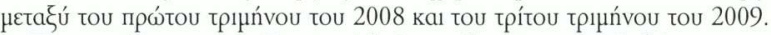

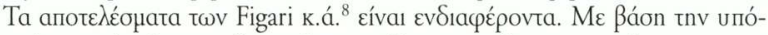

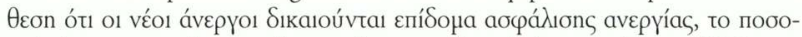

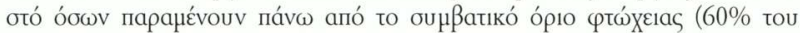

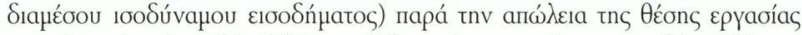

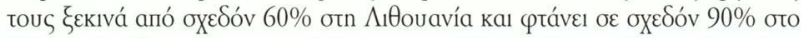

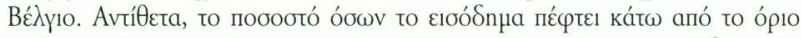

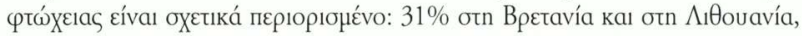

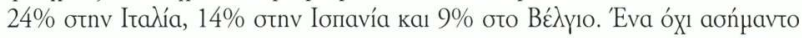

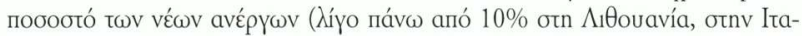

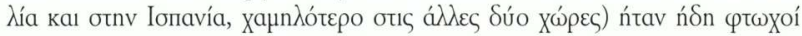

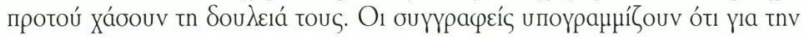

7. H. Sutherland, «EUROMOD: the tax-benefit microsimulation model for the European Union», бто A. Gupta - A. Harding (впц.), Modelling our future: population ageing, health and aged care. International Symposia in Economic Theory and Econometrics 16 (483-488), Elsevier, Amsterdam 2007.

8. F. Figari - A. Salvatori - H. Sutherland, "Economic downturn and stress testing European welfare systems", ó.п. 


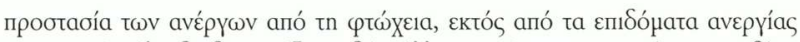

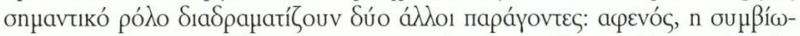

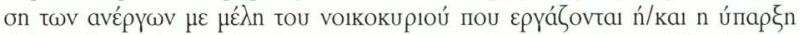

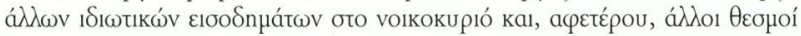

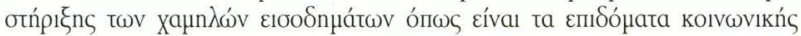

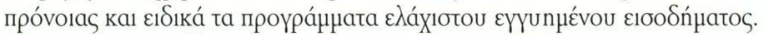

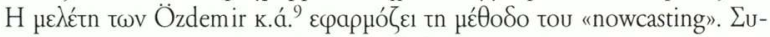

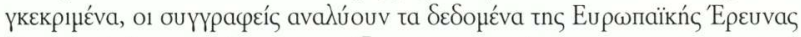

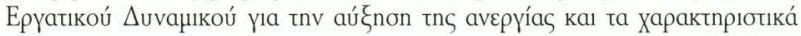

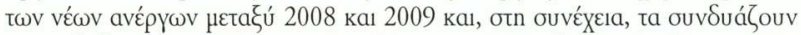

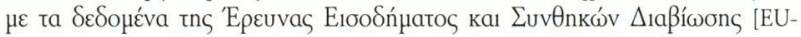

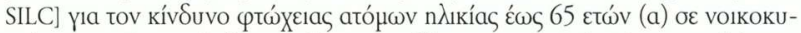

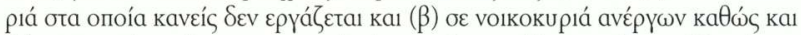

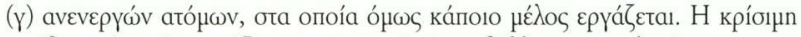

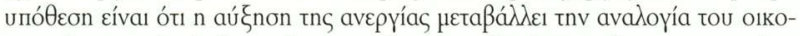

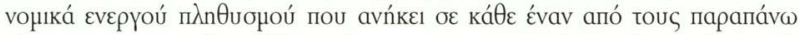

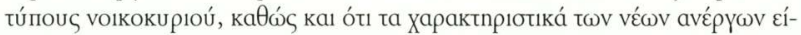

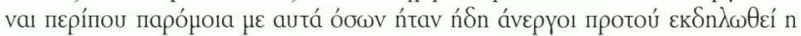

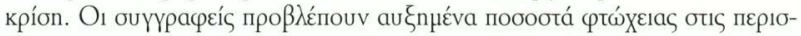

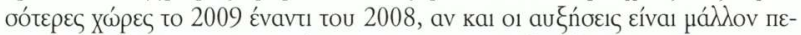

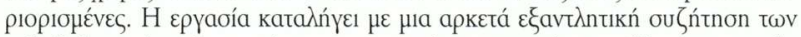

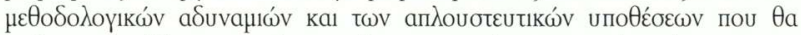

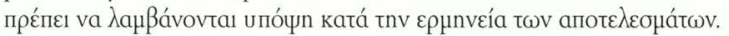

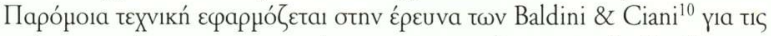

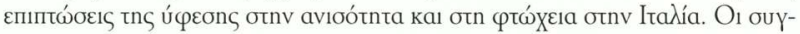

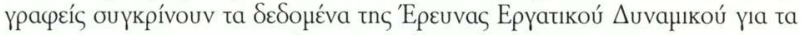

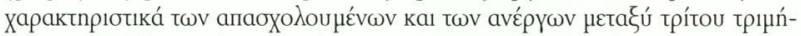

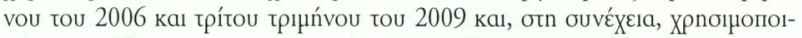

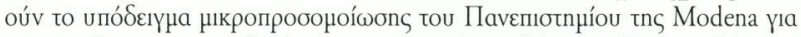

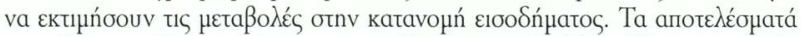

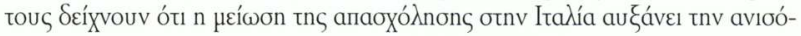

9. E. Ozdemir - F. Sanoussi - T. Ward, «The potential effects of the recession on the risk of poverty: nowcasting", Research note 6/2010, Social Situation Observatory-Income distribution and living conditions, Bpv $\xi \dot{\varepsilon} \lambda \lambda_{\varepsilon \varsigma} 2010$.

10. M. Baldini - E. Ciani, "Diseguaglianza e povertà durante la recessione», CAPPaper $\mathrm{n}$. 75. Università di Modena e Reggio Emilia: Centro di Analisi delle Politiche Pubbliche, Modena 2010. 


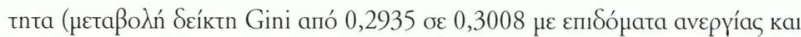

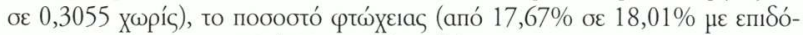

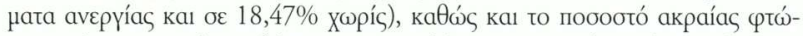

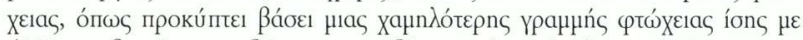

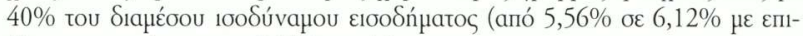

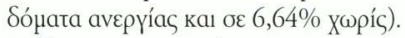

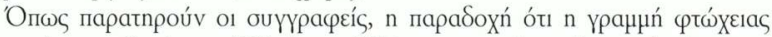

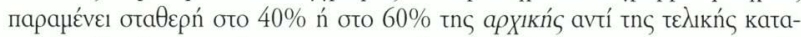

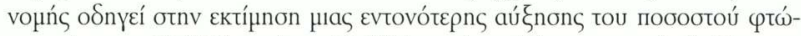

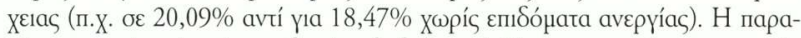

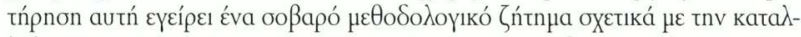

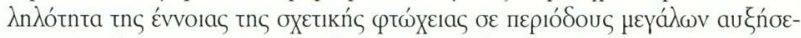

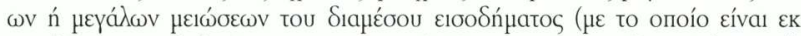

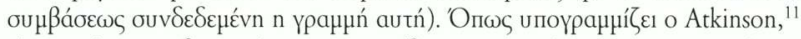

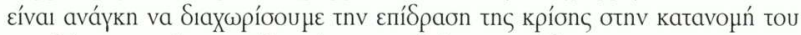

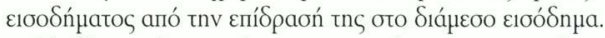

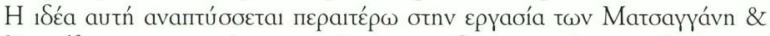

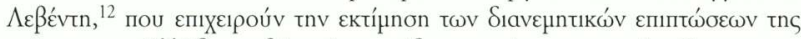

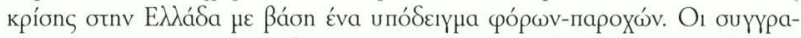

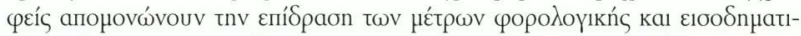

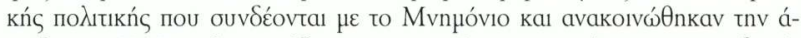

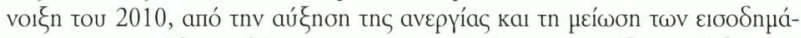

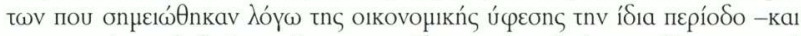

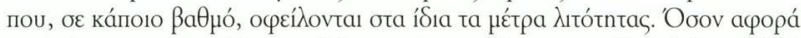

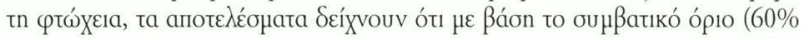

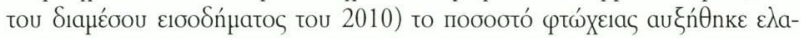

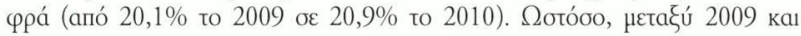

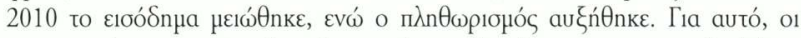

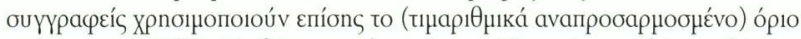

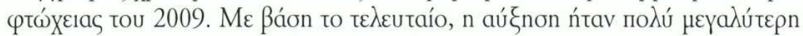
(aпó 20,1\% to 2009 оє 25,1\% to 2010).

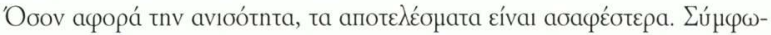

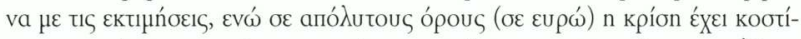

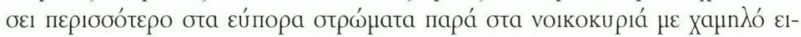

11. A.B. Atkinson, «Stress-testing the welfare state», ó.m.

12. M. Matsaganis - C. Leventi, "Distributional implications of the crisis in Greece», па-

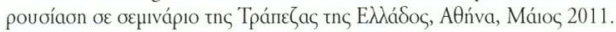




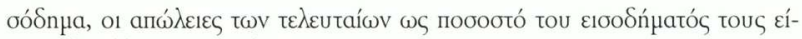

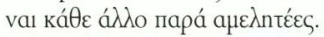

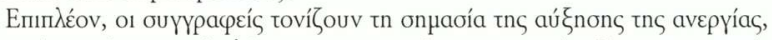

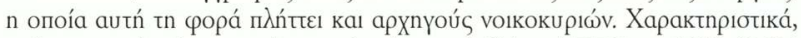

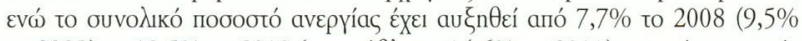

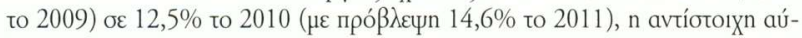

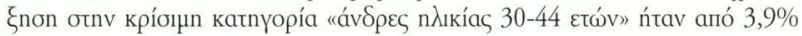
to 2008 бह $8,2 \%$ to 2010 .

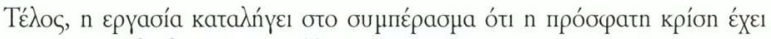

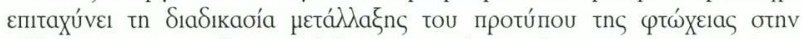

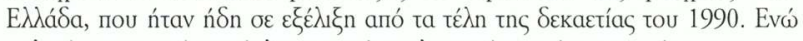

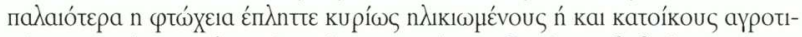

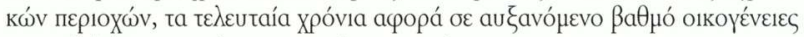

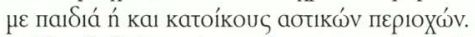

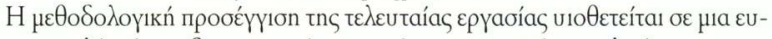

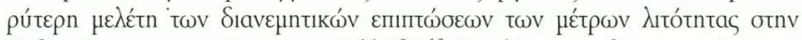

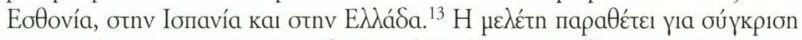

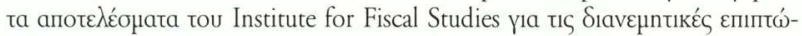

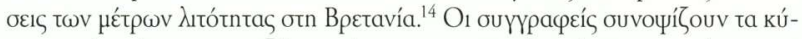

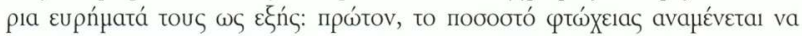

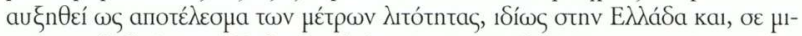

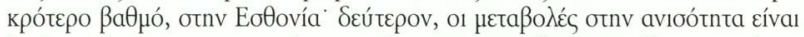

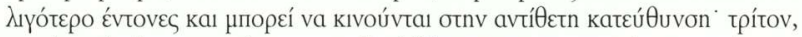

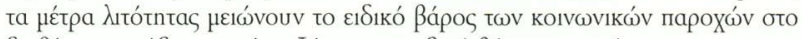

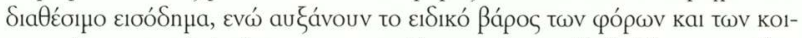

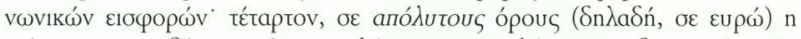

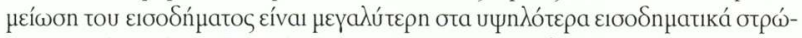

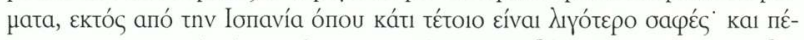

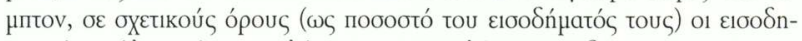

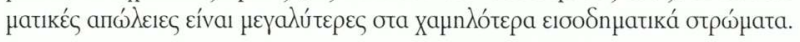

13. C. Leventi - H. Levy - M. Matsaganis - A. Paulus - H. Surherland, "Modelling the distributional effects of austerity measures: the challenges of a comparative perspectiven, Research note 8/2010. Social Situation Observatory-Income distribution and living conditions, Bpu द́̉ $\lambda_{\varepsilon \varepsilon} 2010$.

14. J. Browne - P. Levell, "The distributional effect of tax and benefit reforms to be introduced between June 2010 and April 2014: a revised assessment), IFS Briefing Note

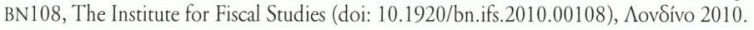




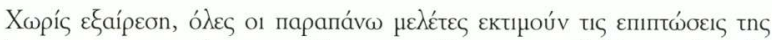

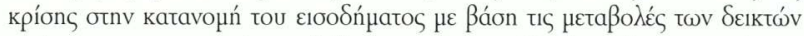

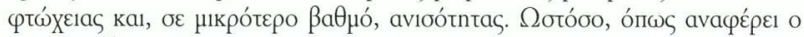

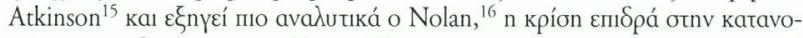

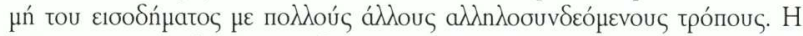

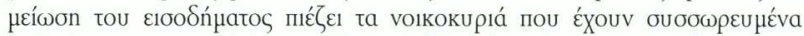

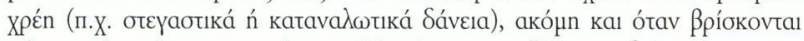

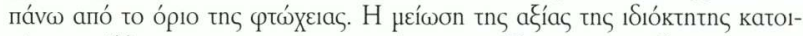

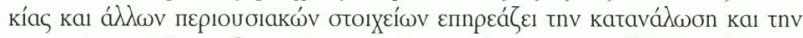

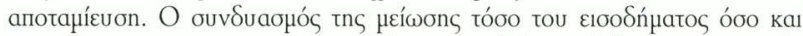

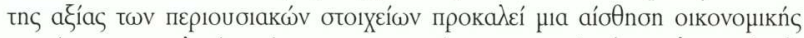

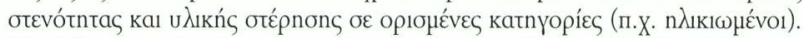

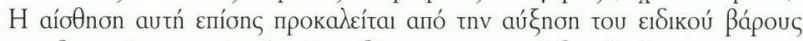

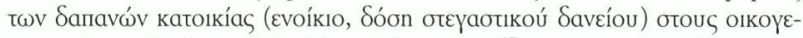

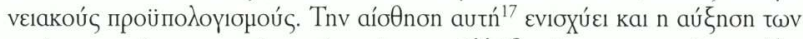

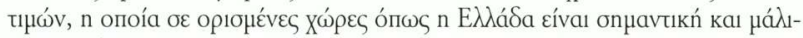

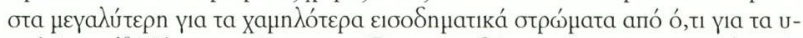

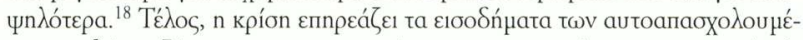

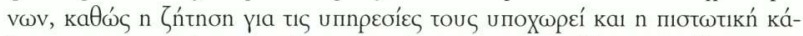

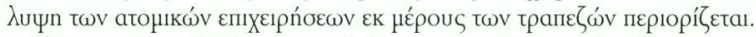

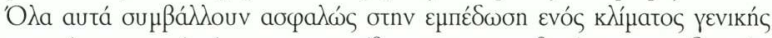

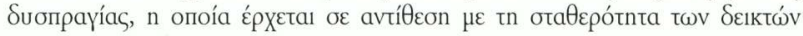

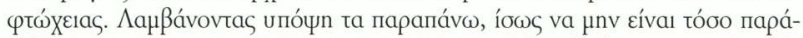

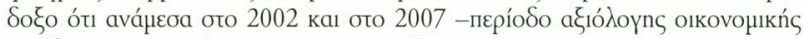

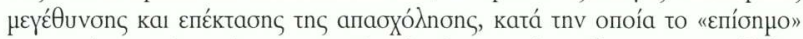

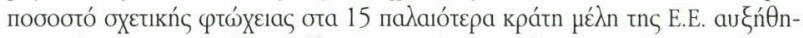

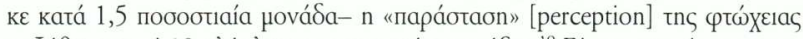

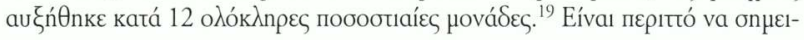

15. A.B. Atkinson, «Stress-testing the welfare state», ó.n.

16. B. Nolan, «Background note for roundtable discussion on monitoring the effects of the financial crisis on vulnerable groups", Organisation for Economic Cooperation and Development, Парі́б 2009.

17. T. Boeri - A. Brandolini, «The age of discontent: Italian households at the beginning of the decade», Giornale degli Economisti, tón. 63, тх. 3-4, 2004, б. 449-487.

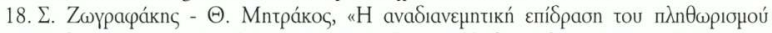

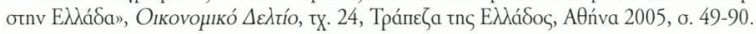

19. Eurostat, Combating poverty and social exclusion: a statistical portrait of the European

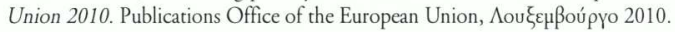




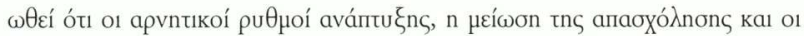

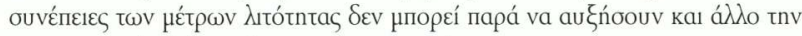

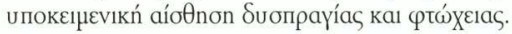

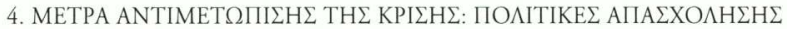

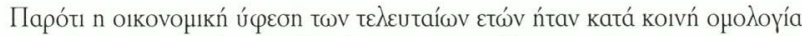

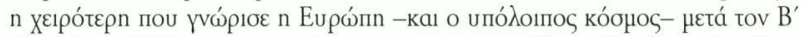

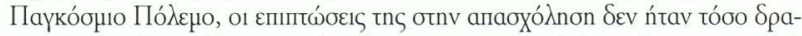

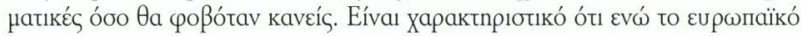

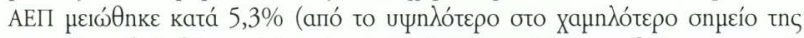

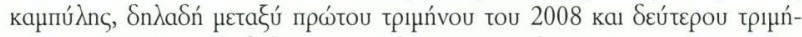

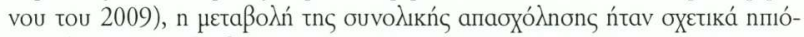

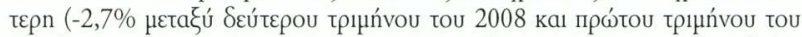

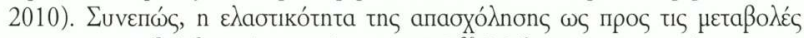

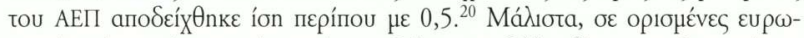

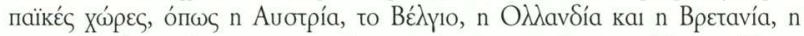

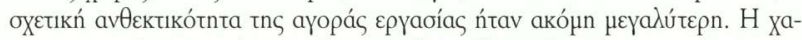

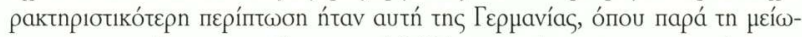

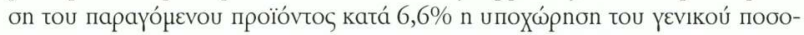

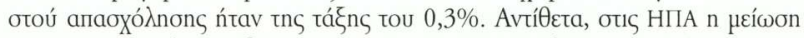

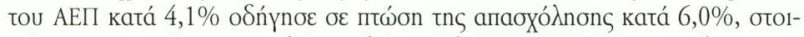

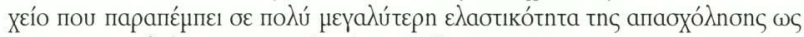

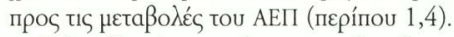

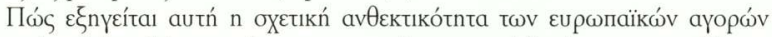

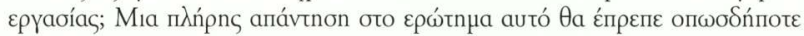

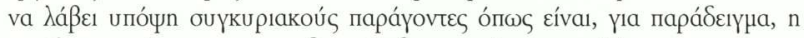

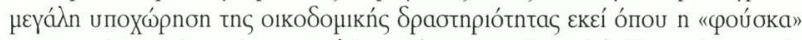

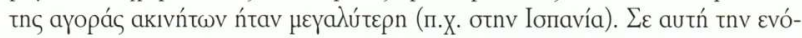

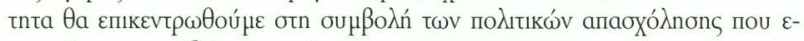

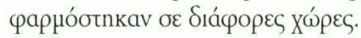

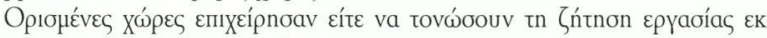

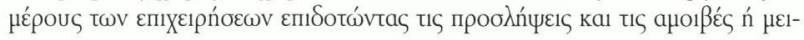

20. EC, Directorate-General for Employment, Social Affairs and Equal Opportunities, Employment in Europe 2010, ó.n. 


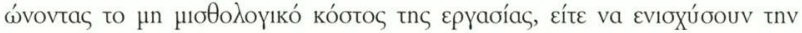
апаоХó yaóías.

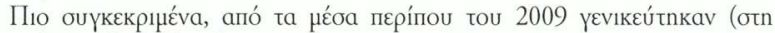

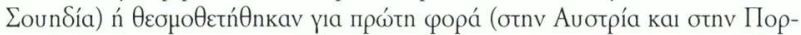

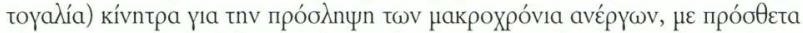

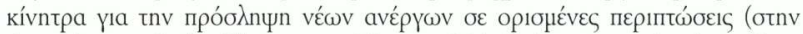

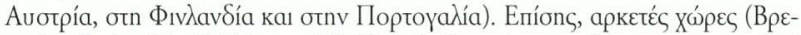

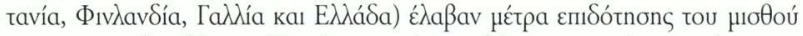

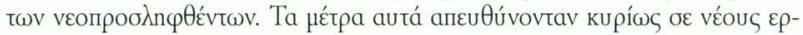

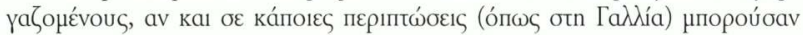

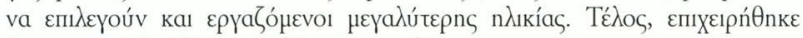

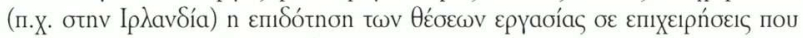

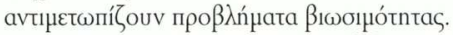

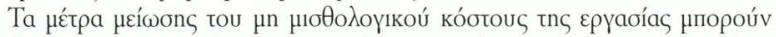

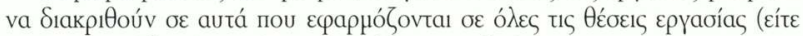

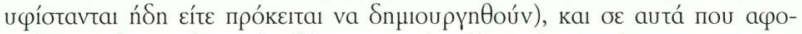

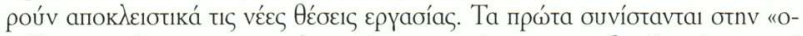

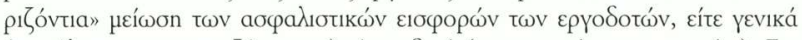

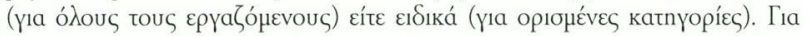

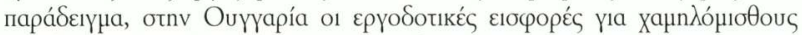

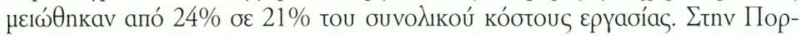

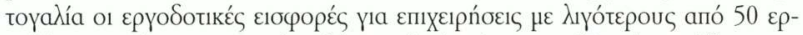

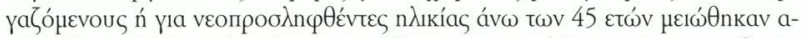

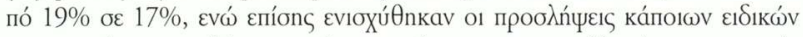

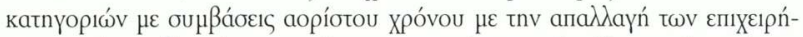

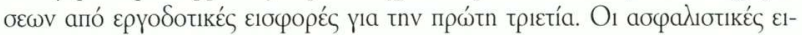

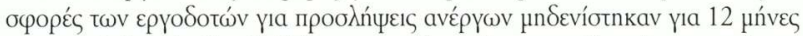

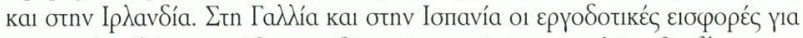

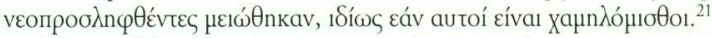

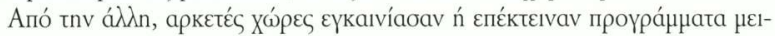

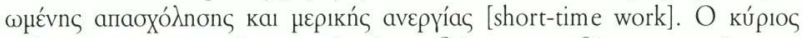

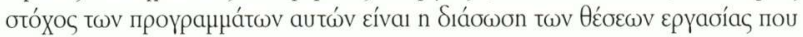

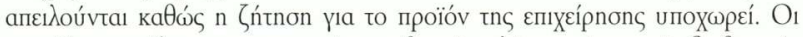

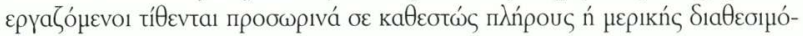

21. OECD, Employment outlook: moving beyond the jobs crisis, ó.n. 


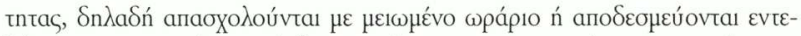

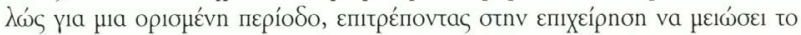
kóotos $\mu$ 1。

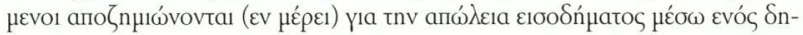

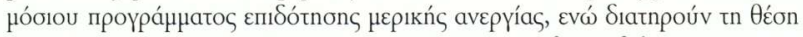

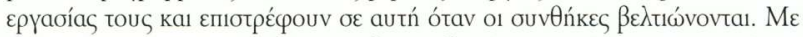

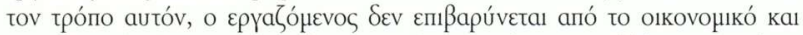

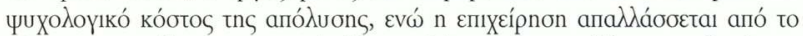

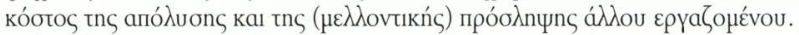

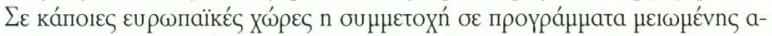
пабХó

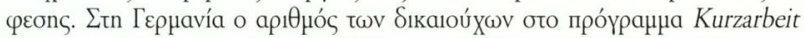

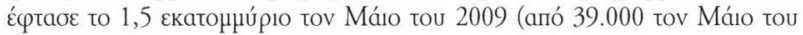

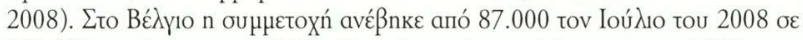
313.000 tov Máptio tou 2009. Etnv Auбtpía tov Aúyouбto tou 2008 про-

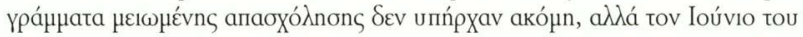

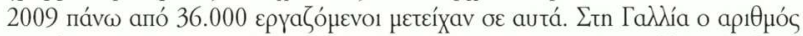

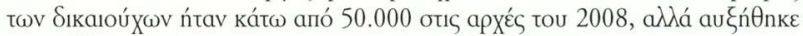

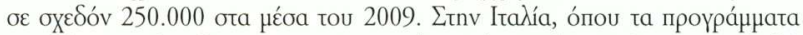

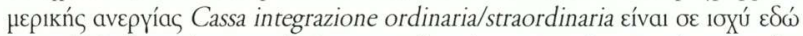

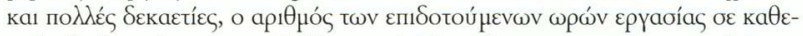

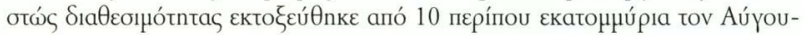

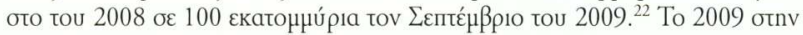

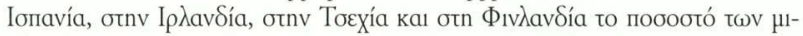

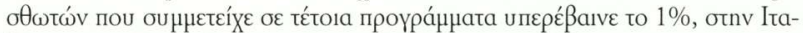

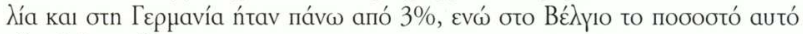

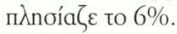

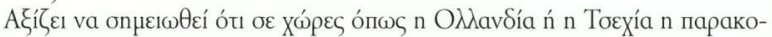

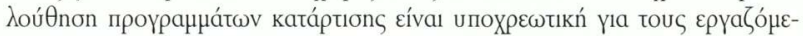

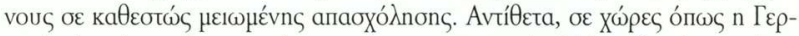

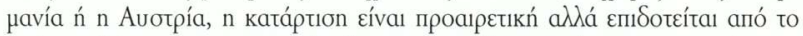

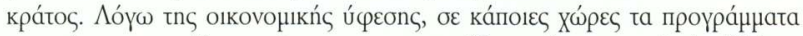

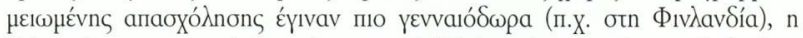

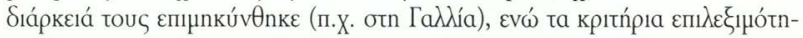

22. EC, Directorate-General for Employment, Social Affairs and Equal Opportunities, Employment in Europe 2010, ó.n. 


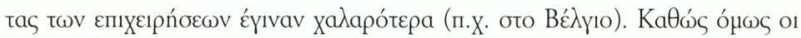

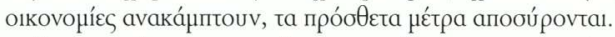

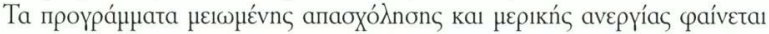

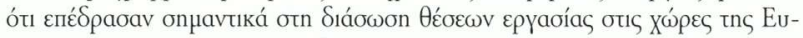

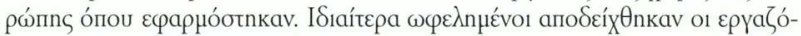

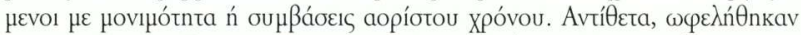

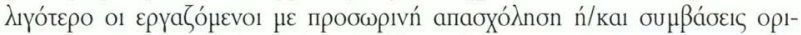

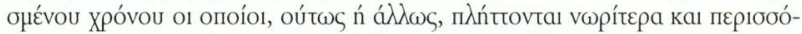

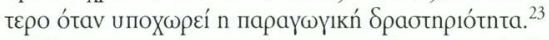

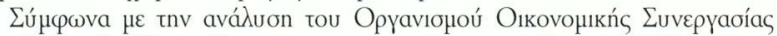

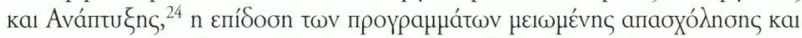

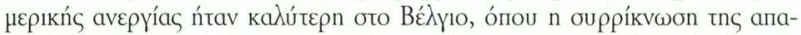

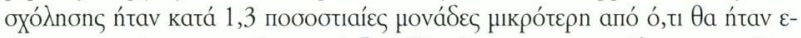

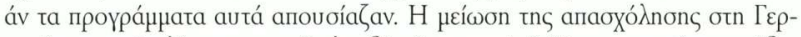

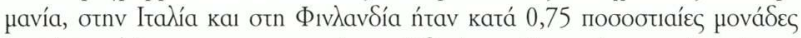

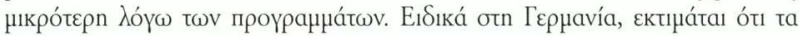

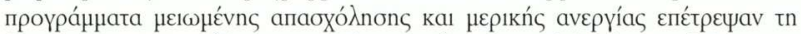

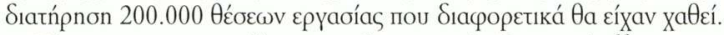

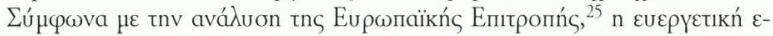

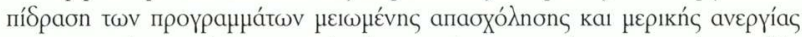

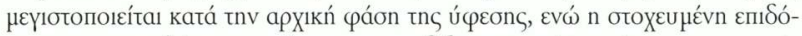

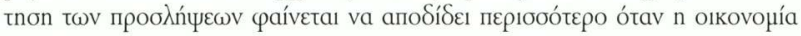

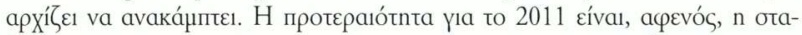

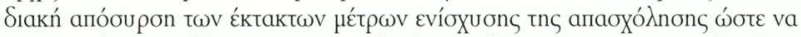

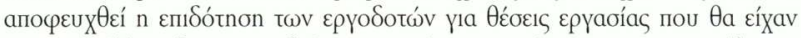

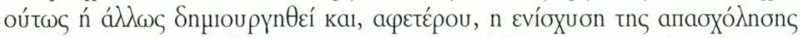

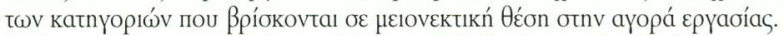

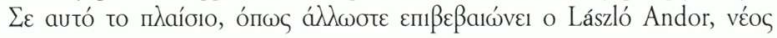

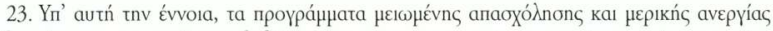

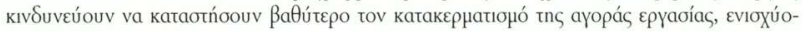

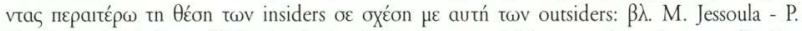
Graziano - I. Madama "Selective flexicurity" in segmented labour markets: the case of Italian "mid-siders" ", Journal of Social Policy, tóp. 39, тx. 4, 2010, o. 561-583 B. Palier (впи.), A long goodbye to Bismarck: the politics of welfare reforms in continental Europe, Amsterdam

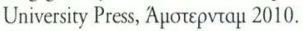

24. OECD, Employment outlook: moving beyond the jobs crisis, ó.n.

25. EC, Directorate-General for Employment, Social Affairs and Equal Opportunities, Employment in Europe 2010, ó.r. 


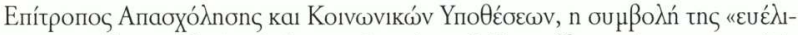

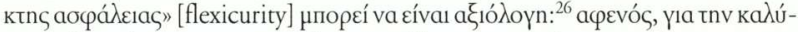

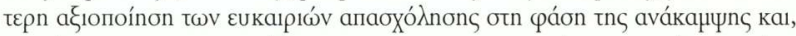

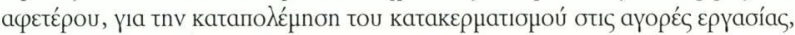

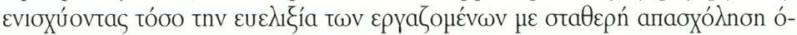

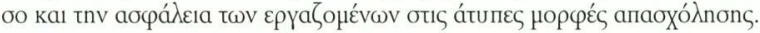

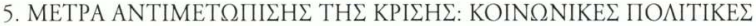

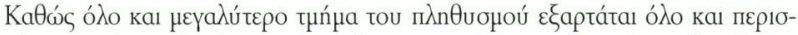

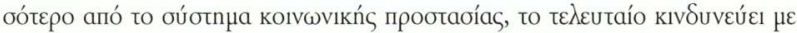

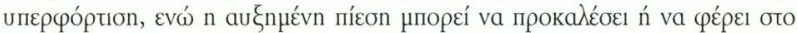

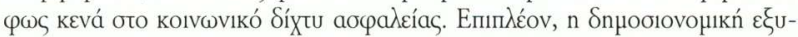

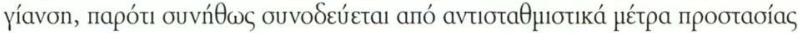

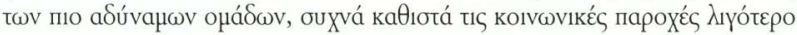

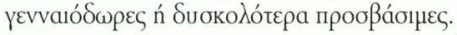

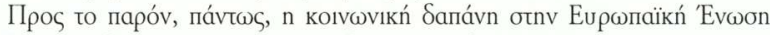

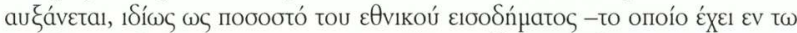

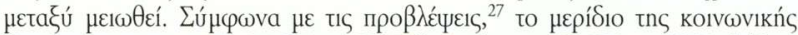

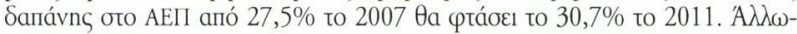

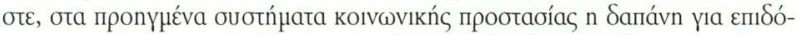

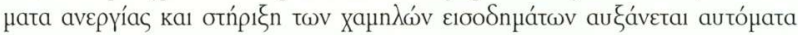

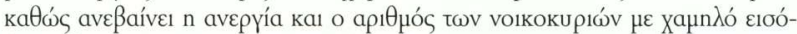

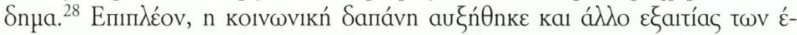

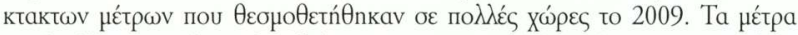

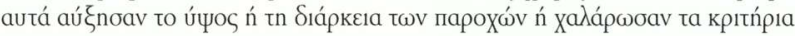

26. B $\lambda$. EC, Directorate-General for Employment, Social Affairs and Equal Opportunities,

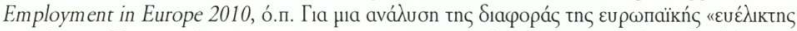

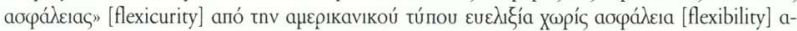

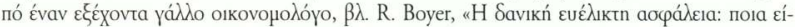

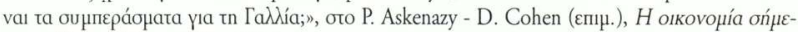

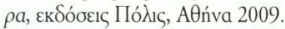

27. CEU, "Update of the Joint Assessment by the Social Protection Committee and the European Commission of the social impact of the economic crisis and of policy responses, 26 November 2010", Council of the European Union, Bpu $\xi^{\prime} \lambda \lambda \varepsilon \varsigma 2010$.

28. B. Vis - K. van Kersbergen - T. Hylands, "To what extent did the financial crisis intensify the pressure to reform the welfare state?», Social Policy \& Administration, unó éxסoon. 


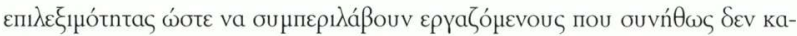

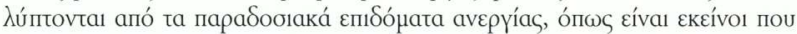

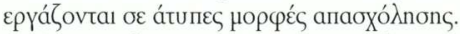

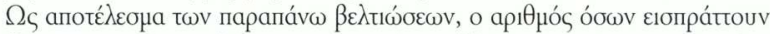

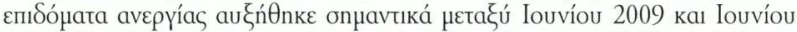

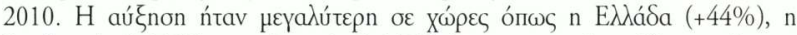

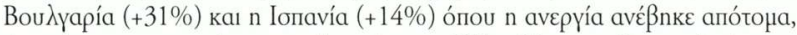

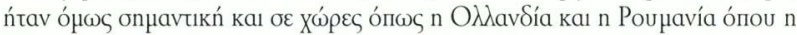

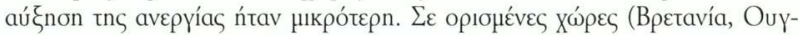

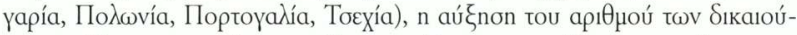

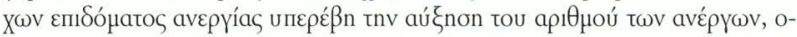

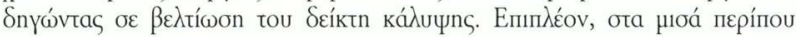

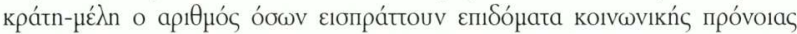

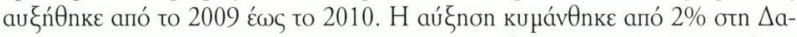

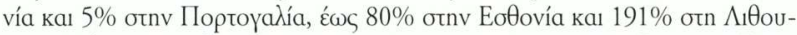

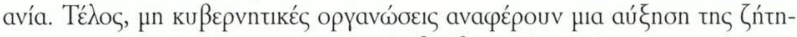

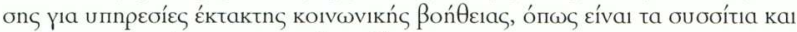

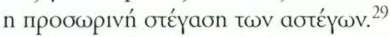

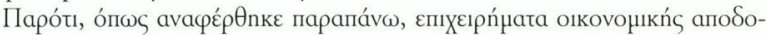

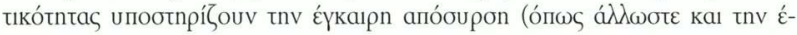

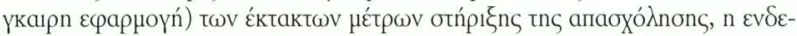

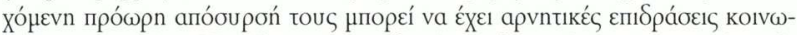

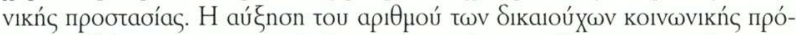

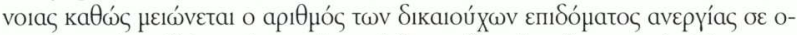

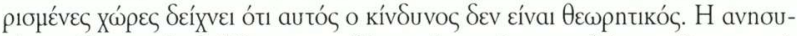
Xía үıа $\delta$ u

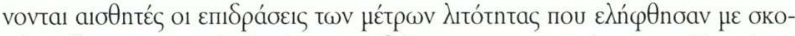

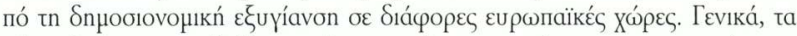

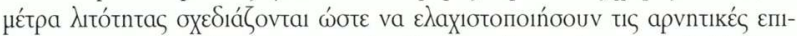

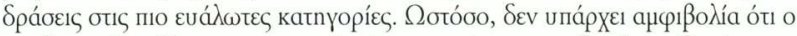

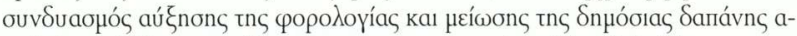

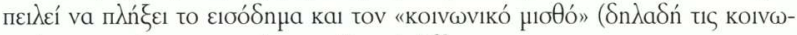

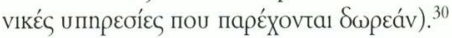

29. CEU, "Update of the Joint Assessment by the Social Protection Committee and the European Commission of the social impact of the economic crisis and of policy responses, 26 November 2010 ", ó.r.

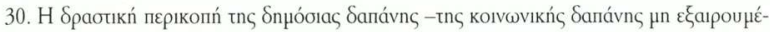




\section{6. ГYMПЕРАГMATA}

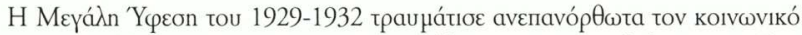

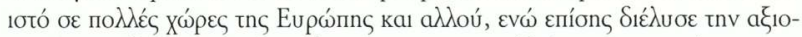

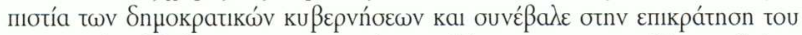

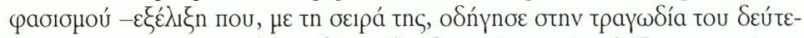


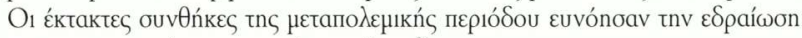

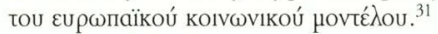

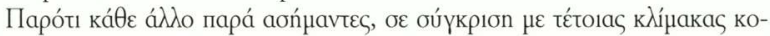

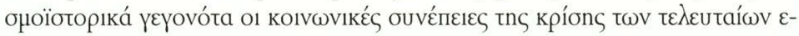

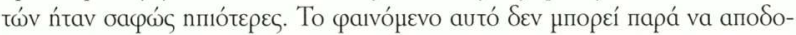

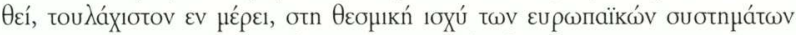

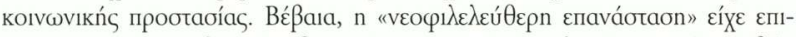

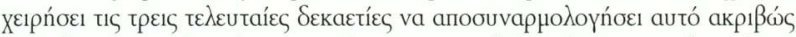

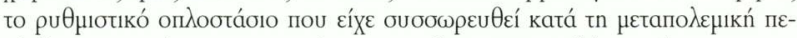

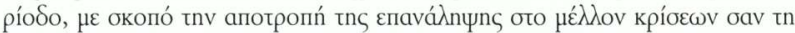

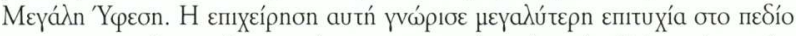

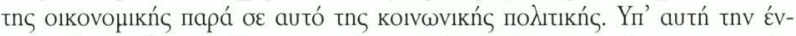

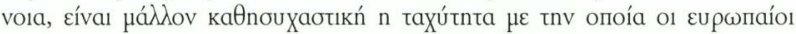

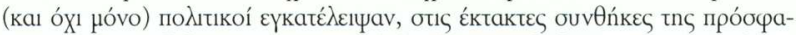

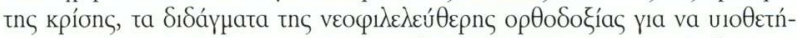

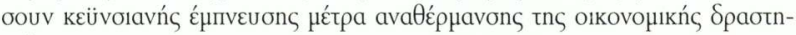
piótntas.

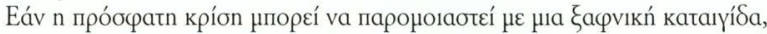

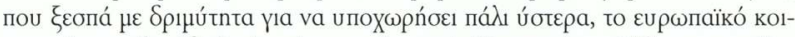

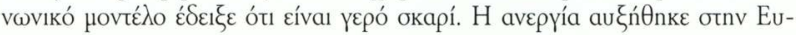

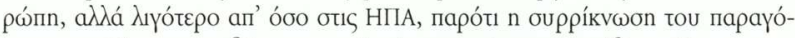

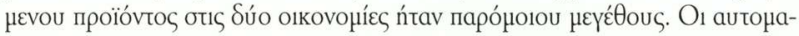

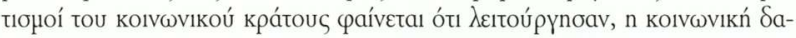

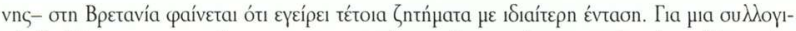

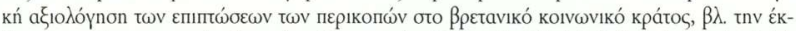

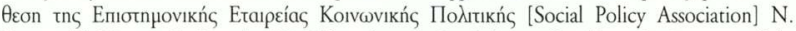
Yeates - T. Haux - R. Jawad - M. Kilkey (впц.), In defence of welfare: the impacts of the spending review. Social Policy Association, www.social-policy.org.uk, 2011.

31. F.G. Castles, "Black swans and elephants on the move: the impact of emergencies on the welfare state», Journal of European Social Policy, tóp. 20, тx. 2, 2010, б. 91-101. 


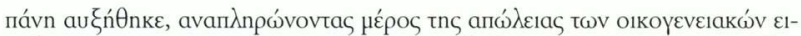

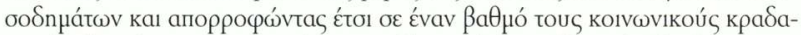

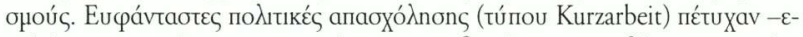

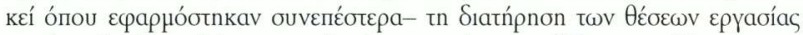

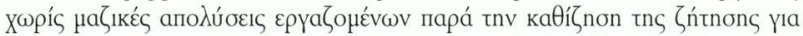

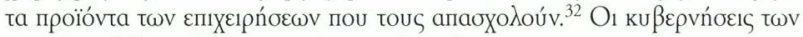

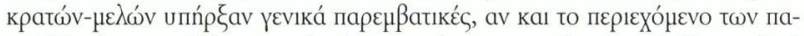

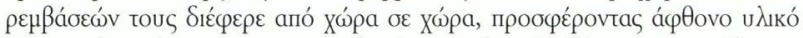

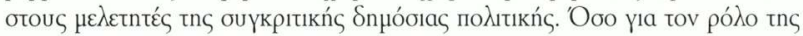

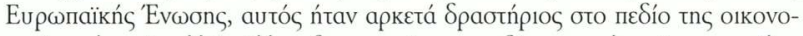

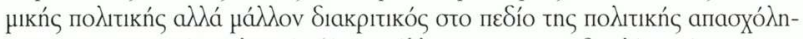

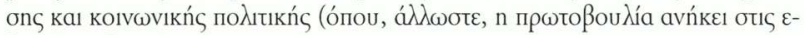

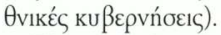

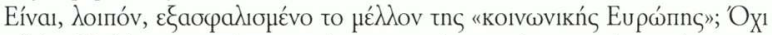

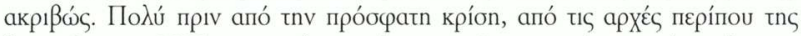

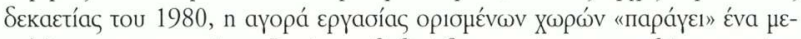

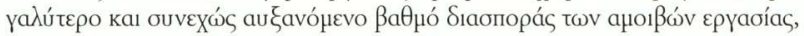

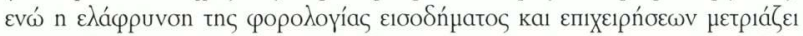

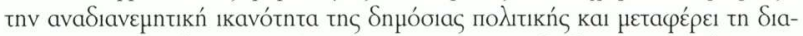

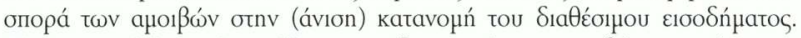

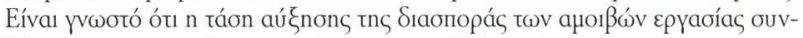

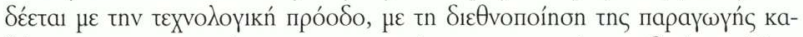

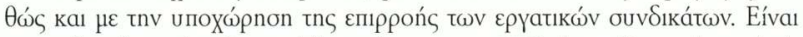

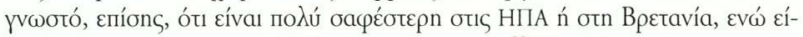

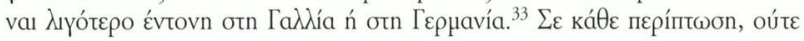

32. European Restructuring Monitor- ERM, Extending flexicurity: the potential of shorttime working schemes, European Foundation for the Improvement of Living and Working Conditions, $\triangle$ oußגívo 2010.

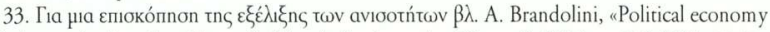
and the mechanics of politics", Politics \& Society, tóp. 38, тx. 2, 2010, o. 212-226 OECD, Growing unequal? income distribution and poverty in OECD countries. Organisation for

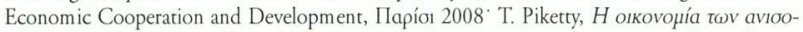

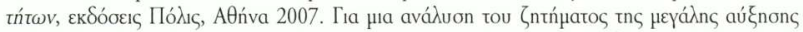

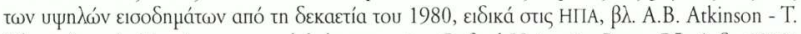

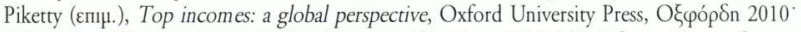

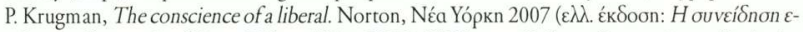

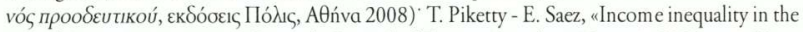
United States, 1913-1998", Quarterly Journal of Economics, tóp. 118, тx. 1, 2003, o. 1-39. 


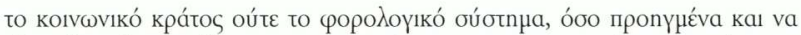

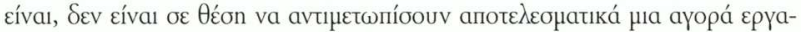

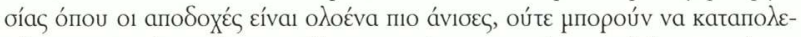

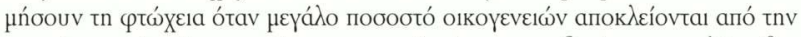

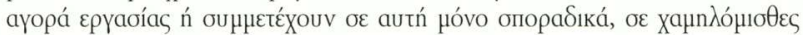

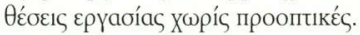

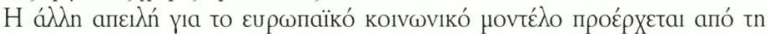

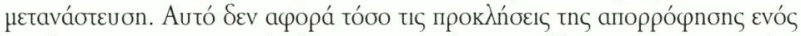

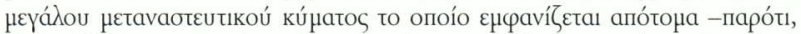

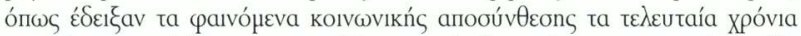

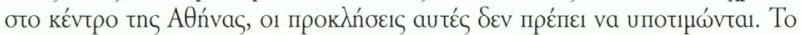

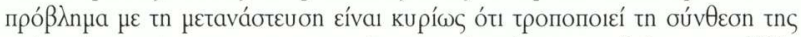

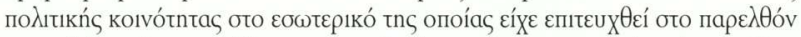

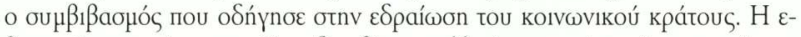

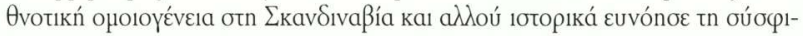

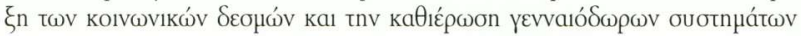

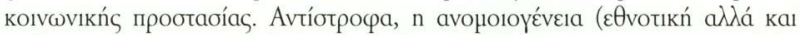

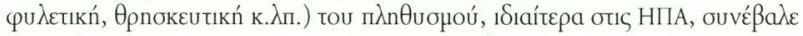

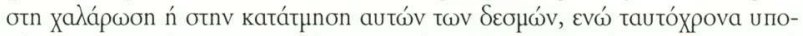

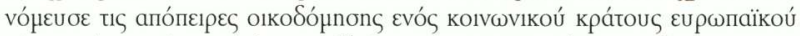

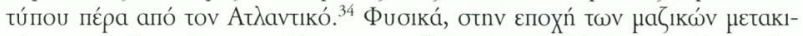

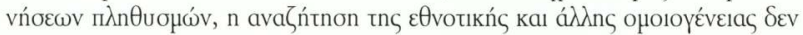

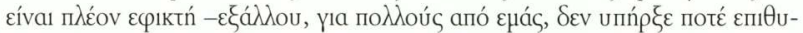

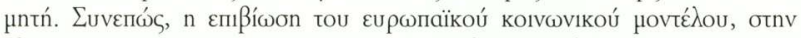

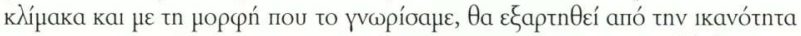

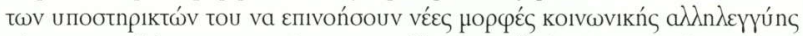

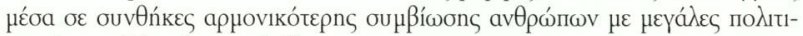

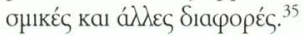

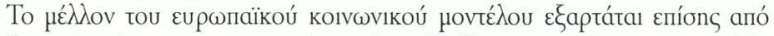

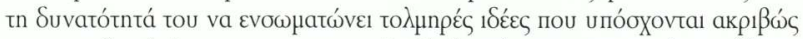

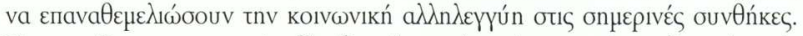

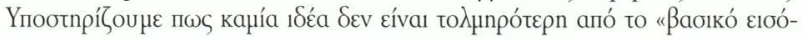

34. A. Alesina - E.L. Glaeser, Fighting poverty in the US and Europe: a world of difference,

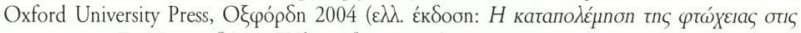

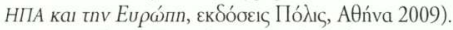

35. A. Glyn, Capitalism unleashed: finance, globalization, and welfare, Oxford University Press, Ȯ̧ópón 2006. 


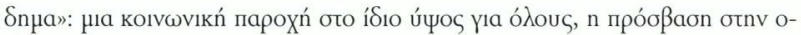

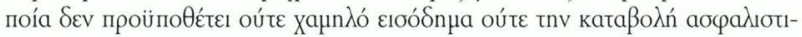

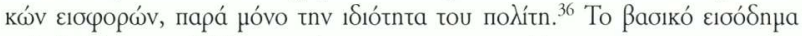

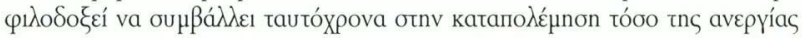

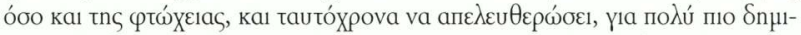

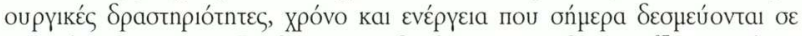

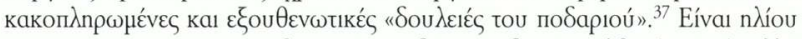

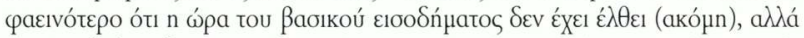

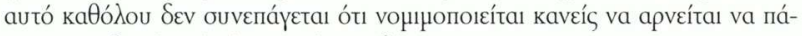

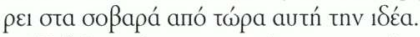

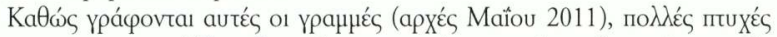

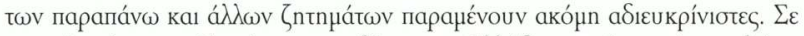

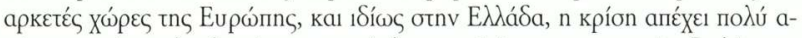

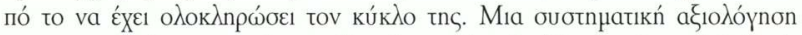

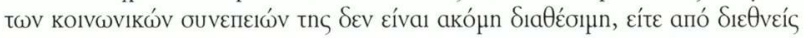

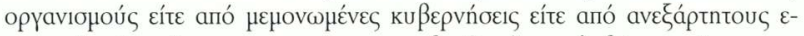

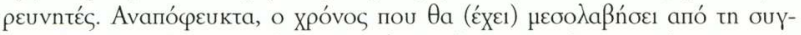

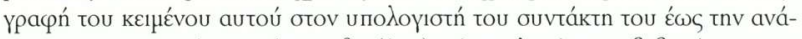

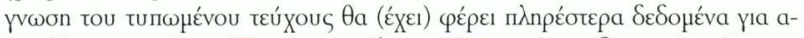

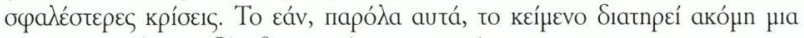

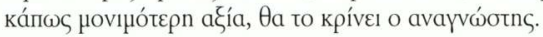

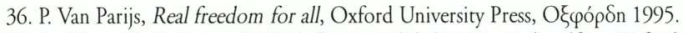

37. A. Glyn, Capitalism unleashed: finance, globalization, and welfare, Oxford University

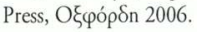

